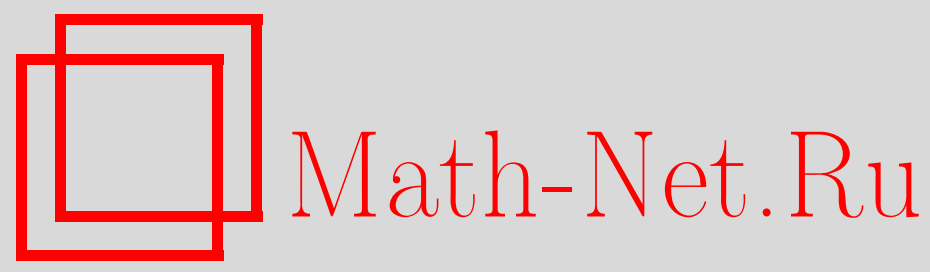

В. П. Маслов, А. М. Чеботарев, Логарифмическая асимптотика решения задачи о больших уклонениях для уравнения Больцмана с малой передачей импульса, Матем. заметки, 1998, том 64, выпуск 1, 73-94

DOI: https://doi.org/10.4213/mzm1374

Использование Общероссийского математического портала Math-Net.Ru подразумевает, что вы прочитали и согласны с пользовательским соглашением http://www . mathnet.ru/rus/agreement

Параметры загрузки:

IP : 18.207 .199 .55

26 апреля 2023 г., 12:28:09

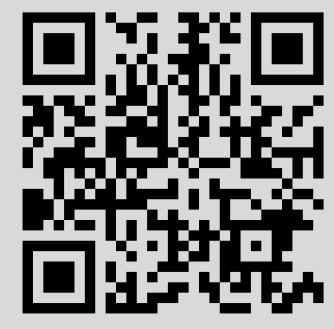




\title{
ЛОГАРИФМИЧЕСКАЯ АСИМПТОТИКА РЕШЕНИЯ ЗАДАЧИ О БОЛЬШИХ УКЛОНЕНИЯХ ДЛЯ УРАВНЕНИЯ БОЛЬЦМАНА С МАЛОЙ ПЕРЕДАЧЕЙ ИМПУЛЬСА
}

\author{
В.П. Маслов, А.М. Чеботарёв
}

Для уравнения Болцмана с малой передачей импульса получена система нелинейных интегро-дифференциальных уравнений, описьвающих логарифмическую асимптотику решения задачи Кошш в области, отстоящей от носителя начального условия на расстояние $O(1)$.

Библиографоия: 16 названий.

1. Введение. В теории кинетических уравнений известен факт слабой сходимости при $N \rightarrow \infty$ нормированных решений уравнений, описьвающих эволющию $N$-частичных плотностей распределения для систем с парным взаимодействием, к решениям нелинейных уравнений для одночастичных плотностей. $\mathrm{K}$ основным примерам такой сходимости относится сходимость решений уравнения Лиувилля для $N$-частичной системы с парным взаимодействием порядка $O\left(N^{-1}\right)$ к решению уравнения Власова (см. [1]-[3]), а также сходимость решений уравнения Колмогорова-Феллера для $N$-частичной системы с интенсивностью скачков порядка $O(N)$ к решению уравнения Больцмана (см. [4]-[6]). В настоящей работе мы воспользуемся этим фактом для того, чтобы определить функции Гамильтона различных задач для уравнения Больцмана и построить логарифмическую асимптотику его решений, удовлетворяющую условиям, необходимым для существования действия $S(v, t)$ в асимптотическом разложении вида

$$
\rho_{\varepsilon}(v, t)=\varkappa_{\varepsilon}(t) \rho_{\varepsilon}^{\mathrm{reg}}(v, t) \exp \left(-\frac{S(v, t)}{\varepsilon}\right), \quad \rho_{\varepsilon}^{\mathrm{reg}}=\rho_{0}+\varepsilon \rho_{1}+\cdots,
$$

где $\varkappa_{\varepsilon}(t)$ - нормировочный множитель, обеспечиваюший слабую сходимость решения $\mathrm{k}$ начальному условию при $t \rightarrow 0, \rho_{\varepsilon}^{\text {reg }}(v, t)$ - функция, регулярно зависящая от малого параметра $\varepsilon$, а $S(v, t)$ - действие, удовлетворяющее уравнению типа Гамильтона-Якоби. Действие $S(v, t)$ является главным членом логарифмической асимптотики решения:

$$
S(v, t)=-\lim _{\varepsilon \rightarrow 0} \varepsilon \ln \rho_{\varepsilon}(v, t)
$$

Работа первого автора выполнена при частичной поддержке объединенного проекта фонда INTAS и Российского фонда фундаментальных исследований, грант № 95-91. Работа второго автора выполнена при частичной поддержке фонда INTAS, грант № 96-0698. 
Уравнения, определяющие компоненты асимптотического разложения (1) решения уравнения Больцмана с малой передачей импульса, являются следствием равенства

$$
\begin{aligned}
& \left(\varepsilon \partial_{t}-\partial_{t} S\right) \varkappa_{\varepsilon}(t)\left(\rho_{0}+\varepsilon \rho_{1}+\cdots\right) \\
& \quad=\left(H\left(\rho_{0}+\varepsilon \rho_{1} \mid v, S_{v}^{(1)}\right)+\varepsilon \widehat{H}_{1}\left(\rho_{0} \mid v, S_{v}^{(1)}\right)+O\left(\varepsilon^{2}\right)\right)\left(\rho_{0}+\varepsilon \rho_{1}+O\left(\varepsilon^{2}\right)\right)
\end{aligned}
$$

которое получается при подстановке (1) в уравнение Больцмана. Здесь и далее для градиента функции $S$ используются обозначения $S_{v}^{(1)}=\partial_{v} S=\operatorname{grad}_{v} S$, а операторы $H, \widehat{H}_{1}$ будут описаны ниже (см. правую часть уравнения (36)). Примером гамильтониана, связанного с уравнением Больцмана, является функция

$$
H(\rho \mid v, p)=C \int d w|v-w| \rho(w) \int_{0}^{2 \pi} d \theta \int_{\mathbb{R}} d r|r| e^{-r^{2} / 2}\left(e^{-\lambda r|v-w|\left(p, n_{\theta}\right)}-1\right),
$$

где $\rho \in L_{1}^{+}\left(\mathbb{R}^{3}\right), \lambda, c>0, p, v, w \in \mathbb{R}^{3}, n_{v}=v /|v| \in \mathbb{R}^{3}, n_{\theta}$ - семейство единичных векторов, ортогональных вектору $n_{v-w}$ и параметризованных углом $\theta$. Функции такого вида являются гамильтонианами туннельного типа (см. [7, гл. 9, §1]), для которых условие невырожденности $\operatorname{det} H_{p p}^{(2)}>0$ выполнено, если плотность $\rho$ такова, что для любых конечных $x, v$ и $p$ из $\mathbb{R}^{3}$ квадратичная форма

$$
\left(x, H_{p p}^{(2)} x\right)=\lambda^{2} C \int d w|v-w|^{3} \rho(w) \int_{0}^{2 \pi} d \theta\left(x, n_{\theta}\right)^{2} \int_{\mathbb{R}} d r|r|^{3} e^{-r^{2} / 2-\lambda r|v-w|\left(p, n_{\theta}\right)}
$$

положительно определена. В случае, если $\rho(w)$ сходится к дельта-функции $\delta(w)$, то гамильтониан вырождается, поскольку $v \perp n_{\theta}$ и, следовательно, $\left(v, H_{p p}^{(2)} v\right)=0$. Заметим также, что для гамильтонианов такого вида гиперплоскость $p=0$ в фазовом пространстве $(v, p)$ состоит из точек покоя:

$$
H_{p}^{(1)}(v, 0)=H_{v}^{(1)}(v, 0)=0
$$

Это свойство влечет за собой стационарность точек безусловного минимума действия.

Другое важное свойство гамильтониана (2) следует из его разложения при малых $\lambda$ :

$$
H(\rho \mid v, p)=\lambda^{2} H_{2}(\rho \mid v, p)+O\left(\lambda^{3}\right),
$$

где $H_{2}(\rho \mid v, p)$ совпадает с вейлевским символом нелинейного эллиптического оператора второго порядка, задающего уравнение Ландау-Фоккера-Планка [6].

В отличие от цепочки уравнений линейного метода ВКБ, уравнения для $S$ и $\rho_{k}$ зацепляются с высшими членами:

$$
\begin{aligned}
S: \partial_{t} S(v, t)+ & H\left(\rho_{0} \mid v, S_{v}^{(1)}(v, t)\right)=0 \\
\rho_{0}: \partial_{t} \rho_{0}(v, t)= & \widehat{H}_{1}\left(\rho_{0} \mid v, S_{v}^{(1)}(v, t)\right) \rho_{0}(v, t)+H\left(\rho_{0} \mid v, S_{v}^{(1)}(v, t)\right) \rho_{1}(v, t) \\
& +H\left(\rho_{1} \mid v, S_{v}^{(1)}(v, t)\right) \rho_{0}(v, t),
\end{aligned}
$$

и т.д. Основное наблюдение настоящей работы заключается в том, что уравнения (4), (5) оказьваются не зависящими от $\rho_{1}, \rho_{2}, \ldots$ в двух случаях. 
В первом случае уравнение Гамильтона-Якоби (3), описьвающее показатель экспоненты функции Грина, зависит только от значений $\rho_{0}(v, t)$ в точке $v^{*}(t)=\arg \min S(v, t)$ безусловного минимума действия. Гамильтониан $H(\rho \mid v, p)$ уравнения Больцмана обращается в нуль вместе с градиентом по $v$, если $p=0$. Поэтому

$$
\left.\left(H\left(\rho_{0} \mid v, S_{v}^{(1)}\right) \rho_{1}+H\left(\rho_{1} \mid v, S_{v}^{(1)}\right) \rho_{0}\right)\right|_{S_{v}^{(1)}=0}=0
$$

в уравнении (5). Эволюция безусловного минимума $v^{*}(t)$ определяется из неявного уравнения $S_{v}^{(1)}\left(v^{*}(t), t\right)=0$, т.е.

$$
\dot{v}^{*}=-\left(S_{v v}^{(2)}\left(v^{*}, t\right)\right)^{-1} S_{v t}^{(2)}\left(v^{*}, t\right) .
$$

Из $(3),(4)$ следует, что $S_{v t}^{(2)}\left(v^{*}, t\right)=-H_{v}^{(1)}\left(v^{*}, 0\right)-H_{p}^{(1)}\left(v^{*}, 0\right) S_{v t}^{(2)}\left(v^{*}, t\right)=0$. Это позволяет получить замкнутую систему относительно $\rho_{0}\left(v^{*}, t\right)$ и $S(v, t)$ (теорема 4$)$.

Функции Гамильтона различных задач для нелинейного уравнения, вообще говоря, различны и зависят от начального условия. В случае больших уклонений точки $v$ от носителя начального условия асимптотика решения состоит из двух частей: “внутренней" составляющей $\rho^{\operatorname{int}}(v, t)$ порядка $O(1)$, на носителе которой $S(v, t) \equiv 0$, и “внешней" экспоненциально убьвающей части $\rho^{\operatorname{ext}}(v, t)$, на носителе которой уравнение Гамильтона-Якоби (4) имеет нетривиальное решение. Составляющая $\rho^{\text {int }}(v, t)$ переносится с сохранением нормы в $L_{1}\left(\mathbb{R}^{n}\right)$ вдоль траекторий гамильтоновой системы в области, где $S(v, t) \equiv 0$. Поэтому нелинейное уравнение переноса (5) не зависит от решения уравнения (4) и не содержит высших членов. Более того, в силу свойства (3) гамильтониана $H_{p}^{(1)}(v, 0)=0$ оказьвается, что $\rho^{\text {int }}(v, t)=\rho_{0}(v)+O(\varepsilon)$. После того, как найдено $\rho_{0}^{\text {int }}(v, t)=\rho_{0}(v)$, логарифмическая асимптотика находится из уравнения $(4)$, а точнее из связанной с ним двухточечной вариационной задачи с лагранжианом, соответствуюшим гамильтониану туннельного типа $\mathscr{H}(v, p)=\int_{\Omega} d w \rho_{0}(w) H(v-w, p)$ :

$$
S(v, t)=\min _{\substack{q(t)=v \\ q(0) \in \partial \Omega(t)}} \int_{0}^{t} d \tau \mathscr{L}\left(\rho_{0}^{\mathrm{int}} \mid q(\tau), \dot{q}(\tau), \tau\right)
$$

решение которой удовлетворяет уравнению Гамильтона-Якоби и описывает эволюцию условного минимума двухточечной функции действия $S(v, w, t)$ на множестве траекторий $q(0)=w \in \partial \Omega(t), q(t)=v$. Вариация точки $w \in \partial \Omega(t)$ дает в линейном приближении необходимое условие минимума: $p(0)=S_{w}^{(1)}\left(v, w^{*}, t\right) \perp T \partial \Omega(t)$, полученное в [7] для асимптотики типа больших уклонений для линейных уравнений туннельного типа.

2. Стохастическая гамильтонова система для многочастичного уравнения Колмогорова-Феллера. Рассмотрим марковский процесс в $N$-частичной системе, описьвающий случайное скачкообразное изменение скоростей сталкивающихся частиц. Предположим, что столкновения между $i$-й и $j$-й частищами равновероятны для всех $i$ и $j$, и имеют случайное экспоненциально распределенное время ожидания $\tau_{i j}$, зависящее от относительной скорости частищ $v_{i}-v_{j}$ и случайного "прищельного" параметра $\omega \in S_{3}$ :

$$
\mathrm{P}\left\{\tau_{i j}>t \mid \omega, v_{i}-v_{j}\right\}=\exp \left(-C_{N, \varepsilon} t\left|v_{i}-v_{j}\right| \cdot|\cos \varphi|\right)
$$


где $C_{N, \varepsilon}\left|v_{i}-v_{j}\right|-$ значение интенсивности столкновений $i$-й и $j$-й частиц, $\omega$ - точка на единичной сфере $S_{3} \subset \mathbb{R}^{3}, n_{\omega}-$ соответствующий единичньй вектор, $\varphi=\varphi\left(\omega, v_{i}-v_{j}\right)-$ случайный угол между векторами $v_{i}-v_{j}$ и $n_{\omega}$. Осью сферы $S_{3}$ является вектор $v_{i}-v_{j}$.

Обозначим $v_{i j}=v_{i j}(\omega)=n_{\omega}\left(n_{\omega}, v_{i}-v_{j}\right)$ и будем считать, что "прицельный" параметр $\omega$ определяет не только время ожидания столкновения, но и изменение скорости обеих частиц:

$$
\begin{gathered}
v_{i} \mapsto v_{i}^{\prime}=v_{i}-v_{i j}(\omega), \quad v_{j} \mapsto v_{j}^{\prime}=v_{j}-v_{j i}(\omega), \\
v_{i}=v_{i}^{\prime}+v_{i j}(\omega)=v_{i}^{\prime}-v_{i j}^{\prime}(\omega), \quad v_{i j}^{\prime}(\omega)=n_{\omega}\left(n_{\omega}, v_{i}^{\prime}-v_{j}^{\prime}\right),
\end{gathered}
$$

что соответствует больцмановской модели упругого рассеяния. Таким образом, интенсивность $\left|v_{i j}(\omega)\right|$ парных столкновений между $i$-й и $j$-й частицами зависит от случайного параметра $\omega$, а распределение величины скачка описывается некоторой мерой $m_{\varepsilon}(d \omega, v)$ :

$$
\mathrm{P}\left\{v_{i j}(\omega) \in B\right\}=\int_{\omega: v_{i j}(\omega) \in B} m_{\varepsilon}\left(d \omega, v_{i}-v_{j}\right), \quad B \in \mathscr{B}\left(\mathbb{R}^{3}\right) .
$$

Преобразование скоростей $(7),(8)$ обеспечивает сохранение суммарной энергии и импульса при столкновении двух частиц одинаковой массы. Интенсивность столкновений $C_{N, \varepsilon}\left|v_{i}-v_{j}\right|(6)$ инвариантна относительно преобразования (7), поскольку $\left|v_{i j}(\omega)\right|=$ $\left|v_{i}-v_{j}\right|=\left|v_{i j}^{\prime}(\omega)\right|$. В дальнейшем изложении мы предполагаем, что семейство мер $m_{\varepsilon}$ обладает аналогичным свойством инвариантности:

$$
m_{\varepsilon}\left(d \omega, v_{i}-v_{j}\right)=m_{\varepsilon}\left(d \omega, v_{i}-v_{j}-2 v_{i j}(\omega)\right)=m_{\varepsilon}\left(d \omega, v_{i}^{\prime}-v_{j}^{\prime}\right) .
$$

Распределения (6) и (9) позволяют представить переходную вероятность $P_{N}(v, t+\Delta t \mid \Gamma)$ с точностью до членов порядка $O(\Delta t)^{2}$ как сумму условных вероятностей множества траекторий, не совершающих скачков за время $\Delta t$ и совершающих один скачок за это время. Отсюда следует уравнение Колмогорова-Феллера для переходной вероятности:

$$
\begin{gathered}
\left(\partial_{t}-\widehat{H}_{N, \varepsilon}\right) P_{N}(v, t \mid \Gamma)=0, \quad P_{N}(v, 0 \mid \Gamma)=I_{\Gamma}(v), \\
\widehat{H}_{N, \varepsilon}=C_{N, \varepsilon} \sum_{i \neq j} \widehat{H}_{i j}^{\varepsilon}, \quad \widehat{H}_{i j}^{\varepsilon}=\int m_{\varepsilon}\left(d \omega, v_{i}-v_{j}\right)\left|v_{i j}(\omega)\right|\left(D_{i j}(\omega)-1\right),
\end{gathered}
$$

где $\partial_{v_{i}-v_{j}}=\partial_{v_{i}}-\partial_{v_{j}}$ и $D_{i j}(\omega)=\exp \left(-\left(v_{i j}(\omega), \partial_{v_{i}-v_{j}}\right)\right)$ - композиция операторов сдвига. Таким образом, $v p$-символ псевдодифференциального оператора $\widehat{H}_{N}^{\varepsilon}$ равен

$$
\begin{aligned}
& H_{N}^{\varepsilon}(\boldsymbol{v}, \boldsymbol{p})=C_{N, \varepsilon} \sum_{i \neq j} H_{i j}^{\varepsilon}(\boldsymbol{v}, \boldsymbol{p}), \quad H_{i j}^{\varepsilon}(v, p)=H^{\varepsilon}\left(v_{i}-v_{j}, p_{i}-p_{j}\right) \\
& H^{\varepsilon}(v, p)=\int m_{\varepsilon}(d \omega, v)\left|\left(n_{\omega}, v\right)\right|\left(e^{\left(n_{\omega}, v\right)\left(n_{\omega}, p\right)}-1\right), \quad v_{i}, p_{i} \in \mathbb{R}^{3}
\end{aligned}
$$

Решение уравнения Колмогорова-Феллера можно представить в виде условного математического ожидания относительно вероятностной меры $\mathscr{P} \boldsymbol{v}, t(d \boldsymbol{v}(\tau))$ на цилиндрической $\sigma$-алгебре непрерьвных слева случайных траекторий $\boldsymbol{v}(\tau)$, начинающихся в момент $\tau=t$ в точке $\boldsymbol{v} \in \mathbb{R}^{3 N}$ и приходящих в момент $\tau=0$ в случайную точку $\boldsymbol{v}(0)$ (см. [8]):

$$
\int \mathscr{P}_{v, t}(d \boldsymbol{v}(\tau)) I_{\Gamma}(\boldsymbol{v}(0))=\mathrm{M}_{\boldsymbol{v}, t} I_{\Gamma}(\boldsymbol{v}(0))=P_{N}(\boldsymbol{v}, t \mid \Gamma),
$$


где $I_{\Gamma}(\cdot)$ - индикатор борелевского множества $\Gamma \subseteq \mathbb{R}^{3 N}$. Компоненты $v_{i}(\tau) \in \mathbb{R}^{3}$ траектории скачкообразного марковского процесса являются решением системы стохастических интегральных уравнений

$v_{i}(s)=v_{i}-\int_{(s, t]} \int_{S_{3}} \sum_{j \neq i} \nu_{\varepsilon}\left(d \tau, d \omega \mid v_{i}(\tau)-v_{j}(\tau)\right) n_{\omega}\left(n_{\omega}, v_{i}(\tau)-v_{j}(\tau)\right), \quad 1 \leqslant i, j \leqslant N$,

где $\nu_{\varepsilon}(T, \Omega \mid v)$ - целочисленная пуассонова мера со средним

$$
\mathrm{M} \nu_{\varepsilon}(T, \Omega \mid v)=\operatorname{mes} T C_{N, \varepsilon} \int_{\omega \in \Omega} m_{\varepsilon}(d \omega, v)|(v, n(\omega))|, \quad \Omega \in \mathscr{B}\left(S_{3}\right),
$$

a $n_{\omega}\left(n_{\omega}, v_{i}(\tau)-v_{j}(\tau)\right)$ - случайная величина скачка скорости, соответствующая преобразованиям $(7),(8)$. Приведенная конструкция уравнения для траекторий марковских скачкообразных процессов с экспоненциальньм временем ожидания скачка (6) и распределением величины скачка (9) является стандартной (см. [5], [6], [9]).

Интеграл $\int_{\mathbb{R}} m_{\varepsilon}(d \omega, v)|(v, n(\omega))|=0(|v|)$ имеет смысл интенсивности скачков из точки $v$, поэтому описанный вьше марковский процесс имеет неограниченную интенсивность скачков и неограниченную величину скачка. В классе таких процессов существуют нерегулярные процессы, совершающие бесконечно много скачков за конечное время с положительной вероятностью (см. [10]). Достаточньг условием регулярности скачкообразного марковского процесса с распределением $m_{\varepsilon}\left(d \omega, v_{i}-v_{j}\right)$ величины скачков и интенсивностью скачков

$$
\varkappa(v)=\sum_{i \neq j} \int_{S_{3}} m_{\varepsilon}\left(d \omega, v_{i}-v_{j}\right)\left|\left(n_{\omega}, v_{i}-v_{j}\right)\right|
$$

является неравенство

$$
\widehat{H}_{N, \varepsilon} \varkappa(v) \leqslant c \varkappa(v)
$$

для некоторой постоянной $c \geqslant 0$, т.е.

$$
\begin{aligned}
& \sum_{i \neq j} \int_{S_{3}} m_{\varepsilon}\left(d \omega, v_{i}-v_{j}\right)\left|\left(n_{\omega}, v_{i}-v_{j}\right)\right| \\
& \quad \times\left(\varkappa\left(v_{1}, \ldots, v_{i}-v_{i j}(\omega), \ldots, v_{j}+v_{i j}(\omega), \ldots v_{N}\right)-\varkappa(\boldsymbol{v})\right) \leqslant c \varkappa(\boldsymbol{v})
\end{aligned}
$$

(см. [11]). Учитывая, что $v_{i j}(\omega)=n_{\omega}\left(n_{\omega}, v_{i}-v_{j}\right)$, имеем $\left(n_{\omega}, v_{i}-v_{j}\right)=\left(n_{\omega}, v_{i j}(\omega)\right)$, и в силу $(10)\left(n_{\omega}, v_{i}-v_{j}-2 v_{i j}(\omega)\right)=-\left(n_{\omega}, v_{i}-v_{j}\right)$. Поэтому

$$
\begin{aligned}
\varkappa\left(v_{1}, \ldots, v_{i}-v_{i j}(\omega), \ldots, v_{j}+v_{i j}(\omega), \ldots v_{N}\right)-\varkappa(\boldsymbol{v}) \\
=\left|\left(n_{\omega}, v_{i}-v_{j}-2 v_{i j}(\omega)\right)\right|-\left|\left(n_{\omega}, v_{i}-v_{j}\right)\right|+\sum_{k}\left(\left|\left(n_{\omega}, v_{i}-v_{k}-v_{i k}(\omega)\right)\right|\right. \\
\left.\quad-\left|\left(n_{\omega}, v_{i}-v_{k}\right)\right|+\left|\left(n_{\omega}, v_{k}-v_{j}+v_{k j}(\omega)\right)\right|-\left|\left(n_{\omega}, v_{k}-v_{j}\right)\right|\right) \\
=-\sum_{k}\left(\left|\left(n_{\omega}, v_{i}-v_{k}\right)\right|+\left|\left(n_{\omega}, v_{k}-v_{j}\right)\right|\right) \leqslant 0 .
\end{aligned}
$$

Следовательно, достаточное условие регулярности вьполнено для $c=0$. Оно обеспечивает существование и единственность решения уравнения Колмогорова-Феллера в классе переходных вероятностей [11]. Таким образом, имеет место 
Теорема 1. Если мера $m_{\varepsilon}(d \omega, v)$ удовлетворяет условию (10), то существует единственное решение уравнения Колмогорова-Феллера (11), являющееся переходной вероятностью и определяющее вероятностную меру непрерывного слева скачкообразного марковского прочесса, удовлетворяющего системе стохастических уравнений (13).

Обозначим через $\mathscr{H}_{T}^{\varepsilon}(v, p)$ гамильтониан, порождаемьй введенной выше случайной целочисленной пуассоновой мерой $\nu_{\varepsilon}(T, \Omega \mid v)$ и гамильтонианом $H^{\varepsilon}(v, p)$ :

$$
\mathscr{H}_{T}^{\varepsilon}(v, p)=\int_{S_{3}} \nu_{\varepsilon}(T, d \omega \mid v)\left(e^{\left(n_{\omega}, v\right)\left(n_{\omega}, p\right)}-1\right) .
$$

Из определения пуассоновой меры $\nu_{\varepsilon}(T, d \omega \mid v)$ следует, что среднее значение стохастического гамильтониана не зависит от времени и равно гамильтониану (12):

$$
T^{-1} \mathrm{M} \mathscr{H}_{T}^{\varepsilon}(v, p)=H^{\varepsilon}(v, p) .
$$

Обозначим

$$
\mu_{\varepsilon}(\Omega, v)=\int m_{\varepsilon}(d \omega, v)\left|\left(n_{\omega}, v\right)\right| \chi_{\Omega}(\omega)
$$

и предположим, что производная

$$
\mu_{\varepsilon}^{\prime}(\Omega, v)=\frac{\partial}{\partial v} \mu_{\varepsilon}(\Omega, v) \in \mathbb{R}^{3}
$$

имеет компоненты, абсолютно непрерывные относительно меры $\mu_{\varepsilon}(\Omega, v)$. Далее обозначим через

$$
\varphi_{\varepsilon}(\omega, v)=\frac{d \mu_{\varepsilon}^{\prime}}{d \mu_{\varepsilon}}(\omega, v): S_{3} \times \mathbb{R}^{3} \rightarrow \mathbb{R}^{3}
$$

$\mu_{\varepsilon}$-абсолютно интегрируемую производную Радона-Никодима меры $\mu_{\varepsilon}^{\prime}$ относительно $\mu_{\varepsilon}$. Частные производные стохастического гамильтониана $\mathscr{H}_{T}^{\varepsilon}(v, p)$ равны

$$
\begin{aligned}
\frac{\partial}{\partial p} \mathscr{H}_{T}^{\varepsilon}(v, p) & =\int_{S_{3}} \nu_{\varepsilon}(T, d \omega \mid v) V\left(n_{\omega}, v, p\right), \\
\frac{\partial}{\partial v} \mathscr{H}_{T}^{\varepsilon}(v, p) & =-\int_{S_{3}} \nu_{\varepsilon}(T, d \omega \mid v) P\left(n_{\omega}, v, p\right),
\end{aligned}
$$

где

$$
\begin{gathered}
V\left(n_{\omega}, v, p\right)=\left(n_{\omega}, v\right) n_{\omega} e^{\left(n_{\omega}, v\right)\left(n_{\omega}, p\right)} \\
P\left(n_{\omega}, v, p\right)=\left(n_{\omega}, p\right) n_{\omega} e^{\left(n_{\omega}, v\right)\left(n_{\omega}, p\right)}+\varphi_{\varepsilon}(\omega, v)\left(e^{\left(n_{\omega}, v\right)\left(n_{\omega}, p\right)}-1\right) .
\end{gathered}
$$

Они имеют средние значения, совпадающие с соответствующими частными производными гамильтониана (12), и определяют стохастическую гамильтонову систему с гамильтонианом $\sum_{j \neq i} \mathscr{H}_{T}^{\varepsilon}\left(v_{i}-v_{j}, p_{i}-p_{j}\right)$ :

$$
\begin{aligned}
& v_{i}(s)=v_{i}-\frac{\partial}{\partial p_{i}} \int_{s}^{t} \sum_{j \neq i} \mathscr{H}_{d \tau}^{\varepsilon}\left(v_{i}(\tau)-v_{j}(\tau), p_{i}(\tau)-p_{j}(\tau)\right), \\
& p_{i}(s)=p_{i}+\frac{\partial}{\partial v_{i}} \int_{s}^{t} \sum_{j \neq i} \mathscr{H}_{d \tau}^{\varepsilon}\left(v_{i}(\tau)-v_{j}(\tau), p_{i}(\tau)-p_{j}(\tau)\right) .
\end{aligned}
$$


Стохастическая система (15) описывает марковский процесс в фазовом пространстве, $i$-я компонента которого совершает скачки $v_{i} \rightarrow v_{i}+V_{i j}(\omega), p_{i} \rightarrow v_{i}+P_{i j}(\omega), V_{i j}(\omega)=$ $V\left(n_{\omega}, v_{i}-v_{j}, p_{i}-p_{j}\right), P_{i j}(\omega)=P\left(n_{\omega}, v_{i}-v_{j}, p_{i}-p_{j}\right)$, определенные вьше, где индекс $j$ распределен равномерно на множестве $\{1, \ldots, N\} \backslash\{i\}$, время ожидания скачков имеет распределение (6), а величина скачка определяется значением случайного параметра $\omega$ : $\mathrm{P}\left\{\omega \in B \mid v_{i}-v_{j}\right\}=m_{\varepsilon}\left(B, v_{i}-v_{j}\right)$.

Пусть $\boldsymbol{v}(\tau)=\left(v_{1}(\tau), \ldots, v_{N}(\tau)\right)$ и $\boldsymbol{p}(\tau)=\left(p_{1}(\tau), \ldots, p_{N}(\tau)\right)$ - решение стохастической системы (15). Используя марковское свойство решений стохастического уравнения (15), нетрудно вывести уравнение Колмогорова-Феллера в фазовом пространстве для эволюции функции плотности $\sigma(\boldsymbol{v}, \boldsymbol{p}, t)=\mathbf{M}_{\boldsymbol{v}, \boldsymbol{p}, t} \sigma_{0}(\boldsymbol{v}(0), \boldsymbol{p}(0))$ :

$$
\begin{aligned}
\frac{d}{d t} \sigma(\boldsymbol{v}, \boldsymbol{p}, t)= & C_{N, \varepsilon} \sum_{j \neq i} \int m_{\varepsilon}\left(d \omega, v_{i}-v_{j}\right)\left|v_{i j}(\omega)\right| \\
& \times\left(\sigma \left(v_{1}, \ldots, v_{i}-V_{i j}(\omega), \ldots, v_{j}+V_{i j}(\omega), \ldots, v_{N},\right.\right. \\
& \left.\left.p_{1}, \ldots, p_{i}+P_{i j}(\omega), \ldots, p_{j}-P_{i j}(\omega), \ldots, p_{N}, t\right)-\sigma(v, p, t)\right) .
\end{aligned}
$$

Поскольку интенсивность скачков скорости и их распределение для марковских случайных процессов $v_{i}(s)$ не зависят от текущих координат $p_{j}(s)$, то условие $(14)$ регулярности минимального решения уравнения (15) также выполнено и имеет место

ТЕОРема 2. Если выполнено условие (10), то существует единственное решение уравнения Колмогорова-Феллера (15), являющееся переходной вероятностью и определяющее вероятностную меру непрерывного слева скачкообразного марковского процесса, удовлетворяющего гамильтоновой системе стохастических уравнений (14).

3. Слабые пределы многочастичных уравнений Колмогорова-Феллера. Из свойства (10) меры $m_{\varepsilon}\left(d \omega, v_{i}-v_{j}\right)$ относительно преобразования скоростей $(7)$ следует, что операторы $\widehat{H}_{N}^{\varepsilon}$ и $\widehat{H}_{i j}^{\varepsilon}$ симметричны относительно двойственности между $L_{1}\left(\mathbb{R}^{3 N}\right)$ и $L_{\infty}\left(\mathbb{R}^{3 N}\right), L_{\infty}\left(\mathbb{R}^{3 N}\right)$ и $M\left(\mathbb{R}^{3 N}\right)$, где $M\left(\mathbb{R}^{3 N}\right)$ - банахово пространство мер Радона:

$$
\left(b, \widehat{H}_{N}^{\varepsilon} \rho_{N}\right)=\left(\widehat{H}_{N}^{\varepsilon} b, \rho_{N}\right), \quad\left(b, \widehat{H}_{N}^{\varepsilon} \sigma_{N}\right)=\left(\widehat{H}_{N \varepsilon} b, \sigma_{N}\right),
$$

a $b \in L_{\infty}\left(\mathbb{R}^{3 N}\right), \rho \in L_{1}\left(\mathbb{R}^{3 N}\right), \sigma_{N} \in M\left(\mathbb{R}^{3 N}\right)$,

$$
\left(b, \rho_{N}\right)=\int b(\boldsymbol{v}) \rho_{N} d \boldsymbol{v}, \quad\left(b, \sigma_{N}\right)=\int b(\boldsymbol{v}) \sigma_{N}(d \boldsymbol{v}), \quad \boldsymbol{v} \in \mathbb{R}^{3 N} .
$$

Пусть $\rho_{N}^{\varepsilon}(\boldsymbol{v}, t)$ - положительное нормированное на единицу в $L_{1}\left(\mathbb{R}^{3 N}\right)$ решение задачи Коши (11), отвечающее начальному условию $\rho_{0}(v)$ такому, что

$$
\rho_{N}^{\varepsilon}(\boldsymbol{v}, 0)=\prod_{i=1}^{N} \rho_{0}\left(v_{i}\right), \quad \int \rho_{0}\left(v_{i}\right)\left(1+\left|v_{i}\right|\right)^{4} d v_{i}<\infty, \quad \int \rho_{0}\left(v_{i}\right) d v_{i}=1 .
$$

Элементы пространств $L_{1}\left(\mathbb{R}^{3 N}\right)$ и $L_{\infty}\left(\mathbb{R}^{3 k}\right)$ естественньм образом отождествляются с соответствующими элементами пространств $M\left(\mathbb{R}^{3 N}\right)$ и $L_{\infty}\left(\mathbb{R}^{3 N}\right)$ при $k<N$. Факт слабой сходимости при $N \rightarrow \infty, N C_{N, \varepsilon} \rightarrow C_{\varepsilon}$ решений уравнения Колмогорова-Феллера 
(11), (15) с начальными распределением, удовлетворяющим условию (17), к решению уравнения Больцмана хорошо известен (см. [6]) и состоит в следующем. Для любой гладкой ограниченной функции $f_{k}\left(v_{1}, \ldots, v_{k}\right)$ существует предел

$$
\begin{gathered}
\lim _{N \rightarrow \infty}\left(F_{k}(\cdot), \rho_{N}^{\varepsilon}(\cdot, t)\right)=\int f_{k}(\boldsymbol{v}) \prod_{i=1}^{k} \rho_{\varepsilon}\left(v_{i}, t\right) d v_{i}, \\
F_{k}(v)=\frac{1}{C_{N}^{k}} \sum_{i_{l} \neq i_{m}} f\left(v_{i_{1}}, \ldots, v_{i_{k}}\right), \quad C_{N}^{k}=\frac{k !}{k !(N-k) !}
\end{gathered}
$$

где $\rho_{\varepsilon}(B, t)$ - семейство положительных нормированных вероятностных мер, удовлетворяющих уравнению Больцмана в слабой форме:

$$
\begin{aligned}
\frac{\partial}{\partial t} \int & f_{k}(\boldsymbol{v}) \prod_{i=1}^{k} \rho_{\varepsilon}\left(v_{i}, t\right) d v_{i} \\
= & C_{\varepsilon} \sum_{i \neq j} \int\left(f_{k}\left(v_{1}, \ldots, v_{i}-v_{i j}(\omega), \ldots, v_{j}-v_{j i}(\omega), \ldots, v_{k}\right)-f\left(v_{1}, \ldots, v_{N}\right)\right) \\
& \times m_{\varepsilon}\left(d \omega, v_{i}-v_{j}\right)\left|v_{i j}(\omega)\right| \rho_{\varepsilon}\left(v_{i}, t\right) \rho_{\varepsilon}\left(v_{j}, t\right) d v_{i} d v_{j} .
\end{aligned}
$$

В частности, при $k=1$ слабьм пределом решений уравнений (11) является решение уравнения Больцмана

$$
\begin{aligned}
\partial_{t} \rho\left(v_{1}, t\right)= & C_{\varepsilon} \int d v_{2} \int m_{\varepsilon}\left(d \omega, v_{1}-v_{2}\right)\left|v_{1,2}(\omega)\right| \\
& \times\left(\rho\left(v_{1}-v_{1,2}(\omega), t\right) \rho\left(v_{2}+v_{1,2}(\omega), t\right)-\rho\left(v_{1}, t\right) \rho\left(v_{2}, t\right)\right),
\end{aligned}
$$

а слабым пределом решений уравнения (15) при $N \rightarrow \infty$ является решение уравнения Больцмана в фазовом пространстве:

$$
\begin{aligned}
& \frac{\partial}{\partial t} \sigma\left(v_{1}, p_{1}, t\right)=C_{\varepsilon} \int d v_{2} d p_{2} \int m_{\varepsilon}\left(d \omega, v_{1}-v_{2}\right)\left|v_{1,2}(\omega)\right|\left(\sigma\left(v_{1}-V_{1,2}(\omega), p_{1}+P_{1,2}(\omega), t\right)\right. \\
& \left.\quad \times \sigma\left(v_{2}+V_{1,2}(\omega), p_{2}-P_{1,2}(\omega), t\right)-\sigma\left(v_{1}, p_{1}, t\right) \sigma\left(v_{2}, p_{2}, t\right)\right)
\end{aligned}
$$

которое вырождается в уравнение Больцмана (18) с начальным условием $\sigma_{0}(v, p)=$ $\rho_{0}(v) \delta(p)$. Скачки скорости и импульса $V_{i j}, P_{i j}$ в $(19)$ определяются так же, как и в уравнении (15).

В случае, если скачки $V_{1,2}(\omega), P_{1,2}(\omega)$ малы, главным членом уравнения (16) является уравнение типа Власова-Лиувилля

$$
\dot{\sigma}+C_{\varepsilon}\left(\left\{\sigma, \overline{H_{\sigma}}\right\}+\sigma \overline{\{\sigma, H\}}\right)=0,
$$

где $\sigma=\sigma(v, p, t),\{\cdot, \cdot\}$ - скобка Пуассона,

$$
\begin{gathered}
\overline{H_{\sigma}}(v, p, t)=\int d v_{2} d p_{2} \sigma\left(v_{2}, p_{2}, t\right) H^{\varepsilon}\left(v_{1}-v_{2}, p_{1}-p_{2}\right), \\
\overline{\{\sigma, H\}}=\int d v_{2} d p_{2} \sum_{i=1}^{3}\left(\frac{\partial \sigma\left(v_{2}, p_{2}, t\right)}{\partial v_{2 i}} \frac{\partial H^{\varepsilon}\left(v_{1}-v_{2}, p_{1}-p_{2}, t\right)}{\partial p_{2 i}}\right. \\
\left.-\frac{\partial \sigma\left(v_{2}, p_{2}, t\right)}{\partial p_{2 i}} \frac{\partial H^{\varepsilon}\left(v_{1}-v_{2}, p_{1}-p_{2}, t\right)}{\partial v_{2 i}}\right)
\end{gathered}
$$


$H^{\varepsilon}(v, p)$ - гамильтониан (12).

Комплексньй метод ВКБ и его обобщения [2], [3], [7] позволяют выделить сингулярную часть решения (1), выводящую решение из класса функций при $\varepsilon \rightarrow 0$. При $\varepsilon \rightarrow 0$ семейство мер

$$
\rho_{\varepsilon}(B, t)=\varkappa_{\varepsilon}(t) \int_{B} d v \rho_{\varepsilon}^{\mathrm{reg}}(v, t) \exp \left(-\frac{S(v, t)}{\varepsilon}\right),
$$

где $\varkappa_{\varepsilon}(t)$ - нормировочньй множитель, $\varkappa_{\varepsilon}(t) \exp (-S(v, t) / \varepsilon)$ - сингулярная часть решения, $\rho_{\varepsilon}^{\text {reg }}=\rho_{0}+\varepsilon \rho_{1}+\cdots-$ регулярная часть решения. Как мы увидим ниже, в $L_{\infty}^{\text {loc }}\left(\mathbb{R}^{3}\right)$ для любой гладкой вешественной функции $S(v)$ существует предел

$$
\lim _{\varepsilon \rightarrow 0} \varepsilon C_{\varepsilon} e^{S(v) / \varepsilon} \widehat{H}_{i j}^{\varepsilon} e^{-S(v) / \varepsilon}=H\left(v_{i}-v_{j}, S_{v_{i}}^{(1)}-S_{v_{j}}^{(1)}\right) .
$$

Функция $H(v, p)$ назьвается функиией Гамильтона оператора $\widehat{H}_{N, \varepsilon}$ с малой передачей импульса. Аналогичньм образом определяется оператор переноса $\widehat{L}$, соответствующий $\widehat{H}_{N, \varepsilon}$ :

$$
\begin{gathered}
\lim _{\varepsilon \rightarrow 0}\left(C_{\varepsilon} e^{S(v) / \varepsilon} \widehat{H}_{i j}^{\varepsilon} e^{-S(v) / \varepsilon}-\varepsilon^{-1} H\left(v_{i}-v_{j}, S_{v_{i}}^{(1)}(v)-S_{v_{j}}^{(1)}(v)\right)\right) \varphi(v)=\widehat{L}_{i j} \varphi(v), \\
\widehat{L}_{i j}=L\left(S^{(2)}(v), v_{i}-v_{j}, \widehat{p}_{i}-\widehat{p}_{j}\right), \quad \widehat{p}_{i}=-\partial_{v_{i}} .
\end{gathered}
$$

Функция $H(v, p)$ и оператор $\widehat{L}(\sigma, v, p)$ зависят от предельных свойств семейства мер $m_{\varepsilon}(d \omega, v)$, которые обсуждаются в следующем пункте.

4. Распределение скачков в случае малой передачи импульса. Для описания динамики системы $N$ частиц, столкновения которых происходят с малой передачей импульса, будем считать, что меры $m_{\varepsilon}(d \omega, v)$ концентрируются при $\varepsilon \rightarrow 0$ вблизи экватора единичной сферы $S_{3}$, осью которой является вектор $n_{v}=v /|v|$. Аналогичное предположение делается при вьводе уравнения Ландау-Фоккера-Планка [6], [12]. Более точно, будем предполагать, что для любой измеримой ограниченной функции $f: \mathbb{R}^{3} \rightarrow \mathbb{R}$ имеет место асимптотическое разложение интеграла:

$$
\int_{S_{3}} m_{\varepsilon}(d \omega, v) f\left(n_{\omega}\left(n_{\omega}, v\right)\right)=\int_{0}^{2 \pi} \frac{d \theta}{2 \pi} \int_{\mathbb{R}} m_{0}(d r \mid v) f\left(\varepsilon r|v| Z\left(\varepsilon r \mid n_{\theta}, n_{v}\right)\right)+O\left(\varepsilon^{\infty}\right),
$$

где $n_{\theta}=(0, \cos \theta, \sin \theta)$ - единичньй вектор, лежащий в плоскости, ортогональной вектору $n_{v}, Z: \mathbb{R} \times S_{3} \times S_{3} \rightarrow \mathbb{R}^{3}$ - гладкая функция, а $m_{0}(d r,|v|)$ - семейство условных вероятностных мер на $\mathbb{R}$. Предположение о концентрации меры $m_{\varepsilon}(d \omega, v)$ вблизи экватора сферы с осью $n_{v}$ означает, что $Z\left(0 \mid n_{\theta}, n_{v}\right)=C n_{\theta}$. Не уменьшая общности, можно считать, что $\left|Z\left(0 \mid n_{\theta}, n_{v}\right)\right|=1$, так как это условие всегда можно обеспечить масштабным преобразованием меры $m_{0}$. Через $\sigma_{k}(v)$ в дальнейшем изложении обозначается конечньй абсолютньй момент меры $m_{0}$ порядка $k$ :

$$
\sigma_{k}(v)=\int_{\mathbb{R}}|r|^{k} m_{0}(d r,|v|) .
$$

Для упрощения доказательств и промежуточных вычислений мы будем дополнительно предполагать, что мера $m_{0}$ симметрична, в частности,

$$
\sigma_{2 k+1}(v)=\int_{\mathbb{R}} r^{2 k+1} m_{0}(d r,|v|)=0,
$$


и что ее первые производные по параметру $v$ имеют не слишком сильные особенности в нуле; так что для любой гладкой ограниченной функции $f(r, v)$, обращающейся в точке $v=0$ в 0 вместе со своей производной первого порядка, первая производная интеграла также равна 0 в точке $v=0$ :

$$
\left.\frac{\partial}{\partial v} \int_{\mathbb{R}} m_{0}(d r,|v|) f(r, v)\right|_{v=0}=0 .
$$

Грубо говоря, условие (24) означает, что первая производная по $v$ меры $m_{0}$ может иметь особенность порядка $O\left(|v|^{-(2-\alpha)}\right), \alpha>0$, в окрестности нуля.

Примером семейства вероятностных мер, удовлетворяющих условиям (22)-(24), является гауссово распределение, дисперсия которого растет с уменьшением скорости и убывает с увеличением температуры:

$$
m_{\varepsilon}(\Omega, v)=\left(\frac{E(v)}{2 \pi k T \varepsilon^{2}}\right)^{1 / 2} \int_{0}^{2 \pi} \frac{d \theta}{2 \pi} \int_{\mathbb{R}} d \varphi \exp \left(-\frac{E(v) \varphi^{2}}{2 \varepsilon^{2} k T}\right) I_{\Omega}(\omega(\theta, \varphi \bmod \pi)),
$$

где $k$ - постоянная Больцмана, $T$ - температура, $E(v)=\frac{1}{2} m v^{2}-$ кинетическая энергия, $I_{\Omega}(\cdot)$ - индикатор борелевского множества $\Omega \subseteq S^{3}, \omega(\theta, \varphi \bmod \pi)$ - точка на сфеpe с угловыми координатами $\theta, \pi / 2+\varphi \bmod \pi$ относительно оси $n_{v}$. Начало отсчета угла $\theta$ выбирается произвольно, точки $\omega(\theta, 0)$ лежат на экваторе сферы с осью $n_{v}$. Обозначим $n_{v}=\{1,0,0\}, n_{\theta}=\{0, \cos \theta, \sin \theta\}, n_{\omega}=\{\sin \varphi, \cos \varphi \cos \theta, \cos \varphi \sin \theta\}$, $n_{v}, n_{\theta}, n_{\omega} \in S_{3} \subset \mathbb{R}^{3}$. Тогда $\left(n_{v}, n_{\theta}\right)=0,\left(n_{\omega}, n_{v}\right)=\sin \varphi, n_{\omega}\left(n_{\omega}, v\right)=\varphi|v|\left(n_{\theta}+\varphi n_{v}+\right.$ $\left.O\left(\varphi^{2}\right)\right)$, так что предположение $(22)$ вьполнено:

$$
\begin{aligned}
\int_{S_{3}} f\left(n_{\omega}\left(n_{\omega}, v\right)\right) m_{\varepsilon}(d \omega, v)= & \left(\frac{E(v)}{2 \pi k T}\right)^{1 / 2} \int_{0}^{2 \pi} \frac{d \theta}{2 \pi} \\
& \times \int_{\mathbb{R}} d \varphi \exp \left(-\frac{E(v) \varphi^{2}}{2 k T}\right) f\left(\varepsilon \varphi|v| Z\left(\varepsilon \varphi \mid n_{\theta}, n_{v}\right)\right)+O\left(\varepsilon^{\infty}\right),
\end{aligned}
$$

где $Z(r)=n_{\theta}+r n_{v}-r^{2} n_{\theta} / 2+O\left(r^{3}\right)$, а член $O\left(\varepsilon^{\infty}\right)$ является оценкой интеграла (25) на множестве $|\varphi| \geqslant \pi$. В этом примере $\left|Z\left(0 \mid n_{\theta}, n_{v}\right)\right|=1, Z\left(0 \mid n_{\theta}, n_{v}\right)=n_{\theta}, Z_{r}^{\prime}\left(0 \mid n_{\theta}, n_{v}\right)=n_{v}$, $Z_{r}^{\prime \prime}\left(0 \mid n_{\theta}, n_{v}\right)=-n_{\theta}$.

Как отмечалось выше, преобразование $v \mapsto v^{\prime}=v-2 n_{\omega}\left(n_{\omega}, v\right)$ является преобразованием зеркального отражения относительно плоскости, ортогональной вектору $n_{\omega}$. Поэтому $|v|=\left|v^{\prime}\right|, E(v)=E\left(v^{\prime}\right)$. Следовательно, мера $m(d \omega, v)$ удовлетворяет условию (10). Для симметричного распределения выполнено предположение (23), и, как нетрудно видеть, первая производная по $v$ меры $m_{0}$ имеет в нуле особенность порядка $O\left(|v|^{-1}\right)$, интегрируемую в двумерном случае. Поэтому предположение (19) также выполнено.

Заметим, что из свойства (17) вытекают равенства

$$
\begin{aligned}
\lim _{\varepsilon \rightarrow 0} \int & m_{\varepsilon}(d \omega, v)\left(n_{\omega}, v\right)^{2}=|v|^{2} \sigma_{2}(|v|), \\
\lim _{\varepsilon \rightarrow 0} \int_{\left|\left(n_{\omega}, v\right)\right| \geqslant \delta|v|} m_{\varepsilon}(d \omega, v)= & \lim _{\varepsilon \rightarrow 0}\left(\frac{E(v)}{2 \pi k T}\right)^{1 / 2} \int_{0}^{2 \pi} \frac{d \theta}{2 \pi} \\
& \times \int_{|r| \geqslant \delta / \varepsilon} d r \exp \left(-\frac{E(v) r^{2}}{2 k T}\right) I_{\Omega}(\omega(\theta, \varphi \bmod \pi))=0,
\end{aligned}
$$


используемые для вывода уравнения Ландау-Фоккера-Планка (см. [6, с. 162]), а меpa (20) имеет абсолютньй момент третьего порядка, равный $\sigma_{3}(v)=O\left(|v|^{-3}\right)$, что соответствует формальной модели кулоновской плазмы [6], [12].

В заключение этого пункта рассмотрим оценку нормировочных коэффициентов $C_{N, \varepsilon}$, обеспечивающих передачу конечной энергии и конечного импульса за единицу времени. Из приведенной выше вероятностной интерпретации уравнений Больцмана и Колмогорова-Феллера (см. [4]-[6]) и разложения (22) следует, что интенсивность столкновений, равна

$$
\begin{aligned}
I_{\varepsilon} & =I_{\varepsilon}(\rho, v)=C_{\varepsilon} \int_{\mathbb{R}} d v^{\prime} \int_{S_{3}} m_{\varepsilon}\left(d \omega, v-v^{\prime}\right)\left|v_{\omega}\right| \rho\left(v^{\prime}\right) \\
& =\varepsilon C_{\varepsilon} \int_{\mathbb{R}} d v^{\prime}\left|v-v^{\prime}\right| \rho\left(v^{\prime}\right)+O\left(\varepsilon^{3}\right) C_{\varepsilon}, \quad v_{\omega}=n_{\omega}\left(n_{\omega}, v-v^{\prime}\right),
\end{aligned}
$$

а средняя величина энергии, передаваемой при парном столкновении равна

$$
\overline{E_{\varepsilon}}(\rho, v)=\frac{m}{2} \frac{\int d v^{\prime} \int_{S_{3}} m_{\varepsilon}\left(d \omega, v-v^{\prime}\right)\left|v_{\omega}\right|^{3} \rho\left(v^{\prime}\right)}{\int d v^{\prime} \int_{S_{3}} m_{\varepsilon}\left(d \omega, v-v^{\prime}\right)\left|v_{\omega}\right| \rho\left(v^{\prime}\right)}=O\left(\varepsilon^{2}\right) .
$$

Поэтому средняя величина импульса, передаваемого при индивидуальном столкновении, имеет порядок $\overline{P_{\varepsilon}}=\sqrt{2 m \overline{E_{\varepsilon}}}$. Из условия передачи конечного импульса $I_{\varepsilon} \overline{P_{\varepsilon}}=O(1)$ мы находим значение нормировочного коэффициента в интеграле столкновений: $C_{\varepsilon}=$ $O\left(\varepsilon^{-2}\right)$. В дальнейшем изложении мы полагаем $C_{\varepsilon}=\varepsilon^{-2}$. Такой выбор нормировочного коэффициента и распределения $(18),(19)$ величины скачков приводит в пределе при $N \rightarrow \infty$ к уравнению Больцмана, описывающему распределение скоростей для столкновительного процесса с частьми столкновениями и малой передачей импульса. Выбор нормировочного коэффищиента $C_{\varepsilon}=\varepsilon^{-3}$ соответствует приближению Ландау-Фоккера-Планка при $\varepsilon \rightarrow 0$.

\section{5. Гамильтониан уравнения Колмогорова-Феллера для многочастичных} систем с малой передачей импульса. Воспользуемся предположением (22) и формулой (12) для асимптотического разложения операторов $\widehat{H}_{i j}^{(\varepsilon)}$ при $\varepsilon \rightarrow 0$. С помощью коммутационного соотношения ПДО с экспонентой (см. [7]) получим выражение для символа операторов $\left.\widehat{H}_{i j}^{(\varepsilon)}\right|_{\varepsilon=0}$ и выражение для оператора переноса, вытекающие из тождества

$$
\begin{aligned}
e^{S(v) / \varepsilon} \widehat{H}_{i j}^{(\varepsilon)} e^{-S(v) / \varepsilon} \rho_{\varepsilon}(v)= & C_{\varepsilon} \int m_{\varepsilon}\left(d \omega, v_{i}-v_{j}\right)\left|\left(n_{\omega}, v_{i}-v_{j}\right)\right| \\
& \times\left(\left(e^{-\Phi_{i j}(v, \omega)}-1\right) \rho_{\varepsilon}(v)+e^{-\Phi_{i j}(v, \omega)} R_{i j}(v, \omega)\right),
\end{aligned}
$$

где

$$
\begin{aligned}
& \Phi_{i j}(v, \omega)=S\left(v_{1}, \ldots, v_{i}-v_{i j}(\omega), \ldots, v_{j}+v_{i j}(\omega), \ldots, v_{N}\right)-S(v), \\
& R_{i j}(v, \omega)=\rho_{\varepsilon}\left(v_{1}, \ldots, v_{i}-v_{i j}(\omega), \ldots, v_{j}+v_{i j}(\omega), \ldots, v_{N}\right)-\rho_{\varepsilon}(v),
\end{aligned}
$$

a $v_{i j}(\omega)=n_{\omega}\left(n_{\omega}, v_{i}-v_{j}\right)$ - определенная вьше функция. Учитывая, что согласно предположению $(22)$ интегралы по семейству мер $\mu_{\varepsilon}$ ограниченных измеримых функций от 
$v_{i j}(\omega)=\left|v_{i}-v_{j}\right| n_{\omega}\left(n_{\omega}, n_{v_{i}-v_{j}}\right)$ сходятся при $\varepsilon \rightarrow 0$ к интегралам по мере $m_{0}$ функций от $\varepsilon v_{i j}(\theta, r)$, где

$$
v_{i j}(\theta, r)=\left|v_{i}-v_{j}\right| Z\left(\varepsilon r \mid n_{\theta}, n_{v_{i}-v_{j}}\right)=\left|v_{i}-v_{j}\right|\left(Z(0)+\varepsilon r Z_{r}^{\prime}(0)\right)+O\left(\varepsilon^{2}\right),
$$

имеем $\Phi_{i j}(v, \omega) \rightarrow \varepsilon \Phi_{\varepsilon}^{i j}(v, r, \theta)$ и $R_{i j}(v, \omega) \rightarrow \varepsilon R_{\varepsilon}^{i j}(v, r, \theta)$, причем $Z(0)=n_{\theta}$,

$$
\begin{gathered}
\Phi_{\varepsilon}^{i j}(v, r, \theta)=\left(-r \widehat{L}_{1}\left(v_{i}-v_{j}, \partial_{v_{i}-v_{j}}\right)+\varepsilon r^{2} \widehat{L}_{2}\left(v_{i}-v_{j}, \partial_{v_{i}-v_{j}}\right)\right) S(v)+O\left(\varepsilon^{2}\right), \\
R_{\varepsilon}^{i j}(v, r, \theta)=r \widehat{L}_{1}\left(v_{i}-v_{j}, \partial_{v_{i}-v_{j}}\right) \rho_{\varepsilon}(v)+O\left(\varepsilon^{1}\right), \\
L_{1}(v, p)=L_{1}\left(v, p \mid n_{\theta}, n_{v}\right)=|v|\left(p, n_{\theta}\right), \\
L_{2}(v, p)=L_{2}\left(v, p \mid n_{\theta}, n_{v}\right)=|v|\left(p, Z_{r}^{\prime}\left(0 \mid n_{\theta}, n_{v}\right)\right)+\frac{1}{2}|v|^{2}\left(p, n_{\theta}\right)^{2} .
\end{gathered}
$$

Нетрудно видеть, что $L_{1}(v, 0)=L_{2}(v, 0)=0$. Заметим также, что в разложениях, отвечающих распределению скачков $(25)$, имеем $L_{1}(v, p)=|v|\left(p, n_{\theta}\right), L_{2}(v, p)=(v, p)+$ $|v|^{2}\left(p, n_{\theta}\right)^{2} / 2$.

Используя разложение модуля суммы векторов $|x+\varepsilon y|=|x|+\varepsilon|x|^{-1}(x, y)+O\left(\varepsilon^{2}\right)$ для $x+\varepsilon y=Z(0)+\varepsilon Z_{r}^{\prime}(0)=Z(\varepsilon r)+O\left(\varepsilon^{2}\right)$ и определение $(22)$ меры $m_{0}$, получим $\widehat{H}_{N}^{(\varepsilon)}=\sum_{i \neq j} \widehat{H}_{i j}^{(\varepsilon)}$, где

$$
\begin{aligned}
\varepsilon e^{S(v) / \varepsilon} & \widehat{H}_{i j}^{(\varepsilon)} e^{-S(v) / \varepsilon} \rho_{\varepsilon}(v)=\left|v_{i}-v_{j}\right| \int_{\mathbb{R}}|r| m_{0}\left(d r,\left|v_{i}-v_{j}\right|\right) \int_{0}^{2 \pi} \frac{d \theta}{2 \pi} \\
& \times\left(\left(e^{r\left|v_{i}-v_{j}\right|\left(n_{\theta}, S_{v_{i}}^{(1)}-S_{v_{j}}^{(1)}\right)}-1\right) \rho_{\varepsilon}(v)\right. \\
& +\varepsilon r e^{r\left|v_{i}-v_{j}\right|\left(n_{\theta}, S_{v_{i}}^{(1)}-S_{v_{j}}^{(1)}\right)}\left(\left(-\left|v_{i}-v_{j}\right|\left(n_{\theta}, \partial_{v_{i}-v_{j}}\right) \rho_{\varepsilon}(v)\right.\right. \\
& \left.\left.+r \rho_{\varepsilon}(v) \widehat{L}_{2}\left(v_{i}-v_{j}, \partial_{v_{i}-v_{j}}\right) S(v)+\rho_{\varepsilon}(v)\left(n_{\theta}, Z_{r}^{\prime}\left(0 \mid n_{\theta}, n_{v_{i}-v_{j}}\right)\right)\right)\right)+O\left(\varepsilon^{2}\right) ;
\end{aligned}
$$

$v p$-символ главной части оператора $\widehat{H}_{i j}^{(\varepsilon)}$ (оператор $\partial_{v}$ действует первым, а умножение на $v$-вторьм) назьвается гамильтонианом уравнения Колмогорова-Феллера с малой передачей импульса:

$$
H(v, p)=|v| \int_{0}^{2 \pi} \frac{d \theta}{2 \pi} \int_{\mathbb{R}} m_{0}(d r,|v|)|r|\left(e^{r|v|\left(p, n_{\theta}\right)}-1\right), \quad v, p \in \mathbb{R}^{3} .
$$

Нетрудно видеть, что поскольку $\left(p, n_{\theta}\right)=\left(|p|^{2}-\left(p, n_{v}\right)^{2}\right)^{1 / 2} \sin \theta$, вьполнено $H(v, p)=$ $h\left(|v|^{2},|p|^{2}-\left(p, n_{v}\right)^{2}\right)$. Соответствующая гамильтонова система имеет законы сохранения $|v|^{2}=$ const, $|p|^{2}-(p, v)^{2} /|v|^{2}=$ const и вполне интегрируема. Функция (28) обладает всеми свойствами гамильтониана туннельного типа [7, гл. 9], кроме свойства невырожденности гессиана по импульсам: $\left(v, H_{p p}^{(2)}(v, p) v\right)=0$. Вырождение снимается при интегрировании по начальному распределению

$$
H(\rho \mid v, p)=\int_{\Omega} d w h\left(|v-w|^{2},|p|^{2}-\left(p, n_{v-w}\right)^{2}\right) \rho(w),
$$

если для любых $p, v, \xi \in \mathbb{R}^{3}$

$$
\int_{\Omega} d v_{0} h_{p}^{(2)}\left(\left|v-v_{0}\right|^{2},|p|^{2}-\left(p, n_{v-v_{0}}\right)^{2}\right) \rho_{0}\left(v_{0}\right)\left(|\xi|^{2}-\left(\xi, n_{v-v_{0}}\right)^{2}\right)>0
$$


ЗАмЕчАниЕ 1. В примере (25) гамильтониан не зависит от $|v|^{2}$ :

$$
\begin{aligned}
H(v, p) & =|v| \int_{0}^{2 \pi} \frac{d \theta}{2 \pi} \int_{\mathbb{R}} d r|r|\left(\frac{E(v)}{2 \pi k T \varepsilon^{2}}\right)^{1 / 2} \exp \left(-\frac{E(v) r^{2}}{2 \varepsilon^{2} k T}\right)\left(e^{-r|v|\left(p, n_{\theta}\right)}-1\right) \\
& =\sqrt{\frac{k T}{m \pi}} \int_{\mathbb{R}} d r|r| e^{-r^{2} / 2} \int_{0}^{2 \pi} \frac{d \theta}{2 \pi}\left(e^{-r\left(p, n_{\theta}\right) \sqrt{k T / m}}-1\right) .
\end{aligned}
$$

Важным свойством гамильтонианов такого вида являются равенства

$$
H(v, 0)=0, \quad H_{v}^{(1)}(v, 0)=H_{p}^{(1)}(v, 0)=0 .
$$

Найденные коэффициенты разложения (27) в общем случае выражаются через производные гамильтониана (28):

$$
\begin{gathered}
\left(H_{p}^{(1)}, \partial_{v}\right)=|v| \int_{0}^{2 \pi} \frac{d \theta}{2 \pi} \int_{\mathbb{R}} m_{0}(d r \mid \theta, v)|r| e^{r|v|\left(p, n_{\theta}\right)}\left(Z_{r}^{\prime}\left(0 \mid n_{\theta}, n_{v}\right), \partial_{v}\right) \\
\operatorname{Tr}\left(H_{p p}^{(2)} S_{v v}^{(2)}\right)=|v|^{3} \int \frac{d \theta}{2 \pi} \int_{\mathbb{R}} m_{0}(d r \mid \theta, v)|r|^{3} e^{r|v|\left(p, n_{\theta}\right)}\left(n_{\theta}, S_{v v}^{(2)} n_{\theta}\right) .
\end{gathered}
$$

Поэтому

$$
\varepsilon e^{S(v) / \varepsilon} \widehat{H}_{i j}^{(\varepsilon)} e^{-S(v) / \varepsilon} \rho_{\varepsilon}(v)=\rho_{\varepsilon}(v) H\left(v_{i}-v_{j}, S_{v_{i}}^{(1)}-S_{v_{j}}^{(1)}\right)+\varepsilon \widehat{L}_{N} \rho_{\varepsilon}(v)+O\left(\varepsilon^{2}\right) .
$$

Дифференциальньй оператор первого порядка $\widehat{L}_{N}=\sum_{i \neq j} \widehat{L}_{i j}$ называется оператором переноса $N$-частичного уравнения Колмогорова-Феллера (см. (21)):

$$
\begin{aligned}
\widehat{L}_{i j}= & H_{p}^{(1)}\left(v_{i}-v_{j}, S_{v_{i}}^{(1)}-S_{v_{j}}^{(1)}\right)\left(\partial_{v_{i}}-\partial_{v_{j}}\right) \\
& +\frac{1}{2} \operatorname{Tr}\left(H_{p p}^{(2)}\left(v_{i}-v_{j}, S_{v_{i}}^{(1)}-S_{v_{j}}^{(1)}\right) \partial_{v_{i}-v_{j}} \otimes \partial_{v_{i}-v_{j}} S(v)\right)+l_{i j} .
\end{aligned}
$$

Поправка $l_{i j}=l\left(v_{i}-v_{j}, S_{v_{i}}^{(1)}-S_{v_{j}}^{(1)}\right)$, присутствующая в $(30)$, связана с разложением распределения скачков и $|Z(\varepsilon r)|$ по малому параметру:

$$
l(v, p)=|v|^{2} \int_{0}^{2 \pi} \frac{d \theta}{2 \pi} \int_{\mathbb{R}} m_{0}(d r,|v|)|r| r\left(\left(e^{r L_{1}}-1\right)\left(n_{\theta}, Z_{r}^{\prime}\right)+e^{r L_{1}}\left(p, Z_{r}^{\prime}\right)\right)
$$

где функция $Z\left(r \mid n_{\theta}, n_{v}\right)$ и еепроизводные по $r$ берутся в точке $r=0, l(0, p)=l(v, 0)=0$, а тензорное произведение градиентов в (30) действует только на $S(v)$. Таким образом, имеет место теорема.

ТЕОРема 3. Если выполнены предположения (22), то гамильтониан $H_{N}$ и оператор переноса $\widehat{L}_{N}$ для $N$-частичного уравнения Колмогорова-Феллера с малой передачей импульса определены формулами (28), (30), (31). 
ЗАмЕчаниЕ 2. Для примера (25) имеем

$$
\left(Z(0), Z^{\prime}(0)\right)=\left(n_{\theta}, n_{v}\right)=0, \quad \int_{\mathbb{R}} d r|r| r \exp \left(-r^{2}\right)=0
$$

Поэтому оператор $\widehat{L}_{N}$ переходит в стандартньй оператор переноса $\widehat{L}_{N}=\sum_{i \neq j} \widehat{L}_{i j}$ с компонентами

$$
\begin{aligned}
\widehat{L}_{i j}= & \left(H_{p}^{(1)}\left(v_{i}-v_{j}, S_{v_{i}}^{(1)}-S_{v_{j}}^{(1)}\right), \partial_{v_{i}}-\partial_{v_{j}}\right) \\
& +\frac{1}{2} \operatorname{Tr}\left(H_{p p}^{(2)}\left(v_{i}-v_{j}, S_{v_{i}}^{(1)}-S_{v_{j}}^{(1)}\right) \partial_{v_{i}-v_{j}} \otimes \partial_{v_{i}-v_{j}} S(v)\right)
\end{aligned}
$$

где $H_{p}^{(1)}$ и $\partial_{v_{i}-v_{j}}-$ векторы из $\mathbb{R}^{3}$, a $H_{p p}^{(2)}$ и $\partial_{v_{i}-v_{j}} \otimes \partial_{v_{i}-v_{j}}-$ матрицы размера $3 \times 3 \mathrm{c}$ коэффициентами

$$
\frac{\partial^{2} H}{\partial p_{k} \partial p_{l}} \quad \text { и } \quad \frac{\partial}{\partial v_{i k}} \frac{\partial}{\partial v_{j l}}
$$

соответственно. Очевидно, что в области, где $S(v, t) \equiv 0$, вьполнено $\widehat{L}_{i j}=0$.

6. Необходимые условия существования асимптотических разложений. Предельньй переход к слабому пределу при $N \rightarrow \infty$ в $N$-частичном уравнении Колмогорова-Феллера (11) с учетом разложения (30) дает следующее уравнение для одночастичной плотности:

$$
\begin{aligned}
e^{S\left(v_{1}, t\right) / \varepsilon} \frac{\partial}{\partial t} e^{-S\left(v_{1}, t\right) / \varepsilon} \rho_{\varepsilon}\left(v_{1}, t\right)=-\frac{1}{\varepsilon} \frac{\partial S\left(v_{1}, t\right)}{\partial t} \rho_{\varepsilon}\left(v_{1}, t\right)+\frac{\partial \rho_{\varepsilon}\left(v_{1}, t\right)}{\partial t} \\
=\varepsilon^{-1} \int d v_{2} e^{-S\left(v_{2}, t\right) / \varepsilon}\left(H\left(v_{1}-v_{2}, S_{v}^{(1)}\left(v_{1}, t\right)-S_{v}^{(1)}\left(v_{2}, t\right)\right)+\varepsilon \widehat{L}_{1,2}\right) \\
\quad \times \rho_{\varepsilon}\left(v_{1}, t\right) \rho_{\varepsilon}\left(v_{2}, t\right)+O(\varepsilon) .
\end{aligned}
$$

Уравнение относительно $S(v, t)$, являющееся уравнением типа Гамильтона-Якоби, включает в себя все члены порядка $O\left(\varepsilon^{-1}\right)$ в разложении $(32)$

$$
\partial_{t} S(v, t)+\rho_{\varepsilon}\left(v^{*}, t\right) H\left(v-v^{*}, S^{(1)}(v, t)\right)=0, \quad v^{*}(t)=\arg \min S(v, t),
$$

а уравнение относительно $\rho_{0}(v, t)$ - все члены порядка $O(1)$.

В этом пункте для вывода уравнения переноса относительно $\rho_{0}(v, t)$ нам дополнительно потребуется лемма о втором члене разложения специального класса интегралов Лапласа, вытекающая из стандартного асимптотического разложения [13]:

$$
\varkappa_{\varepsilon} \int d v e^{-S(v) / \varepsilon} F\left(v-v^{*}, S_{v}^{(1)}(v)\right)=F(0,0)+\varepsilon F_{1}+O\left(\varepsilon^{2}\right)
$$

где $\varkappa_{\varepsilon}=(2 \pi \varepsilon)^{-3 / 2}(\operatorname{det} \sigma)^{1 / 2}-$ нормировочный коэффициент, $\sigma=S^{(2)}\left(v^{*}\right)$ - матрица вторых производных действия в единственной точке невырожденного экстремума $v^{*}$ и $S\left(v^{*}\right)=0$.

Пусть $F(v, p)$ - гладкая функция и $p_{\lambda}(v)=\lambda S_{v}^{(1)}(v), v_{\lambda}=\lambda\left|v-v^{*}\right|$. 
Лемма 1. Если при всех достаточно малых $\varepsilon, \lambda \in \mathbb{R}_{+}$функиии

$$
F_{p p}^{(2)}\left(v_{\lambda}, p_{\lambda}(v)\right), \quad F_{v p}^{(2)}\left(v_{\lambda}, p_{\lambda}(v)\right), \quad F_{v v}^{(2)}\left(v_{\lambda}, p_{\lambda}(v)\right)
$$

абсолютно интегрируемы с весом $\left|v-v^{*}\right| \exp (-S(v) / \varepsilon)$, mo

$$
F_{1}=\frac{1}{2} \operatorname{Tr}\left(\sigma^{-1} h(\sigma)\right)
$$

əде $\sigma=S^{(2)}\left(v^{*}\right), \boldsymbol{h}(\sigma)=\sigma F_{p p}^{(2)} \sigma+\sigma F_{p v}^{(2)}+F_{v p}^{(2)} \sigma+F_{v v}^{(2)}$, причем вторые производные функиии $F$ берутся в точке $v=p=0$.

Нетрудно видеть, что правая часть уравнения относительно $\rho_{0}(v, t)$ содержит, во-первых, члены порядка $O(\varepsilon)$, входящие в подынтегральное выражение (32). Это

$$
\begin{aligned}
r_{0}(v)= & \left(H\left(v_{1}-v_{2}, S_{v}^{(1)}\left(v_{1}, t\right)-S_{v}^{(1)}\left(v_{2}, t\right)\right)\right. \\
& \left.\times\left(\rho_{0}\left(v_{1}\right) \rho_{1}\left(v_{2}\right)+\rho_{1}\left(v_{1}\right) \rho_{0}\left(v_{2}\right)+\widehat{L}_{1,2} \rho_{0}\left(v_{1}\right) \rho_{0}\left(v_{2}\right)\right)\right)\left.\right|_{v_{1}=v, v_{2}=v^{*}} .
\end{aligned}
$$

Опуская для краткости аргумент $t$ и учитывая, что $S_{v}^{(1)}\left(v^{*}, t\right)=0$, получим

$$
r_{0}(v)=-\left((b, \nabla)+\frac{1}{2} f\right) \rho_{0}(v)+G\left(\rho_{0}(v), \rho_{1}(v)\right),
$$

где согласно (30), (31)

$$
\begin{gathered}
b=b\left(v \mid \rho_{0}\right)=\rho_{0}\left(v^{*}\right) H_{p}^{(1)}\left(v-v^{*}, S^{(1)}(v)\right), \\
f=f\left(v \mid \rho_{0}\right)=\rho_{0}\left(v^{*}\right) \operatorname{Tr}\left(H_{p p}^{(2)}\left(v-v^{*}, S^{(1)}(v)\right)\left(S^{(2)}(v)+S^{(2)}\left(v^{*}\right)\right)\right)+l\left(v-v^{*}, S^{(1)}(v)\right), \\
G\left(\rho_{0}, \rho_{1}\right)=\left(\rho_{0}(v) \rho_{1}\left(v^{*}\right)+\rho_{1}(v) \rho_{0}\left(v^{*}\right)\right) H\left(v-v^{*}, S_{v}^{(1)}(v)\right) .
\end{gathered}
$$

Во-вторых, это члены порядка $O(\varepsilon)$ из асимптотического разложения первого члена в интеграле (32) по лемме 1

$$
\begin{gathered}
g(v)=\nu(v) \rho_{0}(v), \quad \nu(v)=\operatorname{Tr}\left(\sigma^{-1} h(\sigma, \rho, v)\right), \\
h(\sigma, \rho, v)=\sigma F_{p p}^{(2)} \sigma+\sigma F_{p v_{2}}^{(2)}+F_{v_{2} p}^{(2)} \sigma+\left.F_{v_{2} v_{2}}^{(2)}\right|_{v_{1}=v, v_{2}=v^{*}, p=0},
\end{gathered}
$$

где $F\left(v_{1}, v_{2}, p\right)=\rho_{0}\left(v_{2}\right) H\left(v_{1}-v_{2}, S_{v}^{(1)}\left(v_{1}\right)-p\right)$. Собирая вместе коэффициенты $(34),(35)$, получаем уравнение, играющее роль уравнения переноса

$$
\dot{\rho}_{0}=-(b, \nabla) \rho_{0}+(g-f) \rho_{0}+G\left(\rho_{0}, \rho_{1}\right) .
$$

Эволюция безусловного минимума $v^{*}(t)$ определяется из неявного уравнения $S_{v}^{(1)}\left(v^{*}(t)\right.$, $t)=0$, т.е. $\dot{v}^{*}=-\left(S_{v v}^{(2)}\left(v^{*}, t\right)\right)^{-1} S_{v t}^{(2)}\left(v^{*}, t\right)$. Но из уравнения Гамильтона-Якоби (33) и свойства гамильтониана $H_{v}^{(1)}\left(v^{*}, 0\right)=0$ следует, что $S_{v t}^{(2)}\left(v^{*}, t\right)=-H_{v}^{(1)}\left(v^{*}, 0\right)-$ $H_{p}^{(1)}\left(v^{*}, 0\right) S_{v t}^{(2)}\left(v^{*}, t\right)=0$. Поэтому $v^{*}(t)=v^{*}(0)$.

Рассмотрим два случая, в которых уравнения (33) и (36) существенно упрощаются. Вырождение коэффициентов $l$ и $G$ этого уравнения в точке $v=v^{*}$ связано с тем, что если $p=\nabla S\left(v^{*}, t\right)=0$, то

$$
\left.H\right|_{v=v^{*}}=0,\left.\quad H_{v}^{(1)}(v, 0)\right|_{v=v^{*}}=0,\left.\quad\{b=l=G\}\right|_{v=v^{*}}=0
$$

(см. замечание 2). Значения $f$ и $F$ в точке $v=v^{*}$ можно найти с помощью леммы 1 :

$$
\begin{gathered}
f\left(v^{*}\right)=\left.f\right|_{v=v^{*}}=2 \rho_{0} \operatorname{Tr}\left(\sigma_{t} H_{p p}^{(1)}(0,0)\right), \\
F(v, 0)=\left.F\right|_{v=v^{*}}=\rho_{0}\left(v^{*}, t\right) \operatorname{Tr}\left(\left(\sigma_{t}\right)^{-1} \boldsymbol{h}\left(\sigma_{t}\right)\right),
\end{gathered}
$$

где $\sigma_{t}=S^{(2)}\left(v^{*}, t\right)$ - матрица вторых производных действия в критической точке. Таким образом, имеет место 
СлЕДСТВИЕ. Для того чтобы решение уравнения Больимана (18) допускало разлохсение (1), необходимо, чтобы функиия $\rho_{t}=\rho_{0}\left(v^{*}, t\right)$ удовлетворяла обыкновенному дифференииальному уравнению

$$
\dot{\rho}_{t}=\frac{1}{2} \rho_{t}^{2} \operatorname{Tr}\left(\left(\sigma_{t}\right)^{-1} h\left(\sigma_{t}\right)-\sigma_{t} H_{p p}^{(2)}(0,0)\right) .
$$

Используя разложение действия в окрестности критической точки

$$
S(v, t)=\frac{1}{2}\left(\left(v-v^{*}\right), \sigma_{t}\left(v-v^{*}\right)\right)+O\left(\left|v-v^{*}\right|^{2}\right), \quad \sigma_{t}=S^{(2)}\left(v^{*}, t\right),
$$

и учитьвая, что

$$
\rho_{t} H\left(v-v^{*}, S_{v}^{(1)}(v)\right)=\frac{\rho_{t}}{2}\left(\left(v-v^{*}, \boldsymbol{h}\left(\sigma_{t}\right)\left(v-v^{*}\right)\right)+O\left(\left|v-v^{*}\right|^{3}\right)\right),
$$

получаем уравнение относительно матрищы вторых производных действия в критической точке: $\dot{\sigma}_{t}+\rho_{t} \boldsymbol{h}\left(\sigma_{t}\right)=0$.

ТЕОремА 4. Пусть для распределения скачков скорости выполнены условия (22)-(26). Тогда для существования разложения (1) решения уравнения Больимана (18) необходимо, чтобы функиии $S(v, t), \sigma_{t}, \rho_{t}$ удовлетворяли уравнению типа Гамильтона-Якоби и системе уравнений типа Рикатти относительно $\sigma_{t}=S_{v v}^{(2)}\left(v^{*}, t\right)$ u $\rho_{t}=\rho_{0}\left(v^{*}, t\right)$ :

$$
\begin{gathered}
\dot{\sigma}_{t}+\rho_{t} \boldsymbol{h}\left(\sigma_{t}\right)=0, \quad \dot{\rho}_{t}-\frac{1}{2} \rho_{t}^{2} \operatorname{Tr}\left(\sigma_{t} H_{p p}^{(2)}-2 H_{p v}^{(2)}-\left(\sigma_{t}\right)^{-1} H_{v v}^{(2)}\right)=0, \\
\frac{\partial}{\partial t} S(v, t)+\rho_{t} H\left(v-v^{*}, S^{(1)}(v, t)\right)=0
\end{gathered}
$$

где $\left.\sigma\right|_{t=0}=S^{(2)}\left(v^{*}\right),\left.\rho\right|_{t=0}=\rho_{0}^{\mathrm{reg}}\left(v^{*}, 0\right)$, а производные гамильтониана берутся $\boldsymbol{в}$ mочке $v=p=0$.

Система уравнений (37) позволяет найти, по крайней мере в малом, значения $\sigma_{t}$ гессиана действия $S(v, t)$ и плотность $\rho_{t}$ вдоль критической траектории $v_{t}^{*}=\arg \min S(v, t)$ и с помощью замены времени получить стандартное уравнение Гамильтона-Якоби для $S(v, t)$.

Второй важньй частный случай - отсутствие экспоненциального затухания решений на носителе начального условия при $t=0: S(v, 0) \equiv 0$. В этом случае $\partial_{t} S(v, t) \equiv 0$, $S(v, t) \equiv 0$, поскольку начало координат является точкой покоя гамильтоновой системы с гамильтонианом (28). Это свойство тривиализует оператор переноса:

$$
\begin{aligned}
\widehat{L}_{\rho} & =\int d v_{2} \widehat{L}_{12} \rho\left(v_{2}\right)=\int d v_{2}\left(H_{p}^{(1)}\left(v_{1}-v_{2}, 0\right), \partial_{v_{1}-v_{2}}\right) \rho\left(v_{2}\right) \\
& =\int d v_{2}\left|v_{1}-v_{2}\right| \int_{0}^{2 \pi} \frac{d \theta}{2 \pi} \int_{\mathbb{R}} m_{0}\left(d r,\left|v_{1}-v_{2}\right|\right)|r|\left(n_{\theta}, \partial_{v_{1}-v_{2}}\right) \rho\left(v_{2}\right)=0
\end{aligned}
$$

поскольку $L_{1}=0$, действие и его производные, а также коэффициенты $g, \nu, f, G$ в (34)-(36) равны 0 (см. замечание 1). Следовательно, имеет место 
ПРЕДЛОЖЕНИЕ. Пусть для распределения скачков скорости выполнены условия (22)-(26). Тогда для существования разложсения (1) решения уравнения Больимана в области, где отсутствует әкспоненииальное затухание решений, необхо$\partial u м$ м чтобы $\rho_{0}(v, t)=\rho_{0}(v)$.

В п. 8 мы используем этот факт для вывода уравнений, описьвающих функцию Грина и решение задачи Коши на расстоянии $O(1)$ от носителя начального условия.

7. Асимптотика функции Грина для уравнения Больцмана. Асимптотика функции Грина линейных эволюционньх уравнений туннельного типа может быть построена с помощью туннельного канонического оператора (см. [7]), сводящего асимптотику в случае особого проектирования лагранжева многообразия к интегрированию решений ОДУ, параметризованных неособыми переменными. В неособом случае асимптотическое разложение функции Грина имеет вид

$$
G_{\varepsilon}(v, w, t)=e^{-S(v, w, t) / \varepsilon}\left(\frac{\operatorname{det} S_{v v}^{(2)}\left(v^{*}, w, t\right)}{(2 \pi)^{3}}\right)^{1 / 2} \sum_{k} \varepsilon^{k} \sigma_{k}(v, w, t),
$$

где $\sigma_{k}(v, w, 0)=\delta_{0, k}$. Рассмотренные вьше уравнения $(37)$ для $S(v, t), \rho_{0}\left(v^{*}, t\right)$ переходят в следующие уравнения для $S(v, w, t), \rho_{0}\left(v^{*}, w, t\right)$ :

$$
\begin{gathered}
\partial_{t} S(v, w, t)+\rho_{0}\left(v^{*}, w, t\right) H\left(v-w, S_{v}^{(1)}(v, w, t)\right)=0, \\
\partial_{t} \rho_{0}\left(v^{*}, w, t\right)+\frac{\rho_{0}\left(v^{*}, w, t\right)^{2}}{2} \operatorname{Tr} \mathscr{H}^{(2)}=0
\end{gathered}
$$

где $\mathscr{H}^{(2)}=H_{p p}^{(2)} S_{v v}^{(2)}\left(v^{*}, w, t\right)-2 H_{p v}^{(2)}-H_{v v}^{(2)}\left(S_{v v}^{(2)}\left(v^{*}, w, t\right)\right)^{-1}$, а вторые производные гамильтониана берутся в точке $v=p=0$.

Оба уравнения упрощаются в шкале времени, связанной со значениями $\rho_{0}\left(v^{*}, w, t\right)$ на экстремали действия $v^{*}(w, t)=\arg \min _{v} S(v, w, t)$. Действительно, относительно переменной

$$
\tau=\int_{0}^{t(\tau)} \rho_{0}\left(v^{*}, w, t\right) d t
$$

уравнения (39) имеют вид

$$
\begin{gathered}
\partial_{\tau} S(v, w, t)+H\left(v-w, S_{v}^{(1)}(v, w, \tau)\right)=0 \\
\partial_{\tau} \rho_{0}\left(v^{*}, w, \tau\right)+\rho_{0}\left(v^{*}, w, \tau\right) \frac{\operatorname{Tr} \mathscr{H}_{p p}^{(2)}}{2}=0
\end{gathered}
$$

где вторые производные гамильтониана берутся в точке $v=p=0$, а действия - в точке $\left(v^{*}, w, \tau\right)$. Теперь (40) - стандартное уравнение Гамильтона-Якоби, а (41) - линейное однородное дифференциальное уравнение. Более того, поскольку точка $p=0$ является инвариантной относительно динамической системы с гамильтонианом $H(v, p)$, то $\arg \min S(v, w, t)=v^{*}=w$ и $S(w, w, t)=0$. Поэтому

$$
\rho_{0}\left(v^{*}, w, \tau\right)=\exp \left(-\frac{1}{2} \int_{0}^{\tau} d \tau\left(H_{p p}^{(2)} S_{v v}^{(2)}-H_{v v}^{(2)}\left(S_{v v}^{(2)}\right)^{-1}\right)\right),
$$

где гамильтониан берется в точке $(0,0)$. 
8. Асимптотика решения в области больших уклонений от носителя начального распределения. Пусть $\varphi: \mathbb{R}^{n} \rightarrow \mathbb{R}$-гладкая функция, $\Omega=\{x: B(x) \leqslant 0\}$ - односвязная область в $\mathbb{R}^{n}$, заданная гладкой вещественной вьпуклой вниз функцией $B: \mathbb{R}^{n} \rightarrow \mathbb{R}$ такой, что

$$
\frac{\partial B(x)}{\partial \vec{n}}>0 \text { на } \partial \Omega,
$$

$\vec{n}=\vec{n}(\omega)$ - внешняя нормаль к границе в точке $\omega, S(x, y)$ - гладкая вьпуклая вниз неотрицательная вещественная функция такая, что для любого фиксированного $x$ невырожденньй минимум $S(x, x)=0$ функции $S(x, y)$ достигается при $y=x$.

Асимптотическое разложение интеграла

$$
I_{\varepsilon}(x)=\int_{\Omega} d x e^{-S(x, y) / \varepsilon} \varphi(x)
$$

во внутренних точках множества $\Omega$ хорошо известно [13]:

$$
I_{\varepsilon}^{\mathrm{int}}(x)=\left.\frac{(2 \pi \varepsilon)^{n / 2}}{\left(\operatorname{det} S_{y y}^{(2)}(x, y)\right)^{1 / 2}}\left(\varphi(x)+\varepsilon \varphi_{1}(x)+o(\varepsilon)\right)\right|_{y=x},
$$

где

$$
\varphi_{1}(x)=\frac{1}{2} \operatorname{Tr}\left(\left(S_{y y}^{(2)}(x, y)\right)^{-1} \varphi_{x x}^{(2)}(x)\right) .
$$

Разложение этого интеграла в точках, лежащих вне замыкания множества $\Omega$ на расстоянии $O(1)$ от гранищы, описывается следующим образом. Предположим, что для любого $x \notin \bar{\Omega}$ существует единственная точка $\omega=\omega(x)=\arg \min _{\omega \notin \bar{\Omega}} S(x, \omega) \in \partial \Omega$ такая, что

$$
S_{\xi}^{(1)}(x, \omega(x))=\left.\left(n(\omega), S_{y}^{(1)}(x, y)\right)\right|_{y=\omega(x)} \neq 0,
$$

где $\eta$ - переменные, описывающие касательную плоскость $T \partial \Omega(\omega)$, а $\xi$ - трансверсальная переменная. Напомним, что необходимым условием существования условного минимума в точке $\omega$ является ортогональность градиента действия $\left.\operatorname{grad}_{y} S(x, y)\right|_{y \in \partial \Omega}$ векторам касательного пространства $T \partial \Omega(\omega)$.

Переходя в интеграле (42) к относительным переменным $\varepsilon^{-1 / 2} \eta+\varepsilon^{-1} \xi, \eta \in \mathbb{R}^{n-1}$, $\xi \in \mathbb{R}_{+}$, и разлагая в ряд функции, регулярно зависящие от $\varepsilon^{1 / 2}$, в ряд Тейлора $I_{\varepsilon}(x)=$ $\sum I_{k}(x) \varepsilon^{k / 2}$, получаем его асимптотическое разложение для $x \in \mathbb{R}^{n} \backslash \bar{\Omega}$.

Лемма 2. Если в точке условного минимума $\omega(x)$ выполнено условие (43), то в точках $x \in \mathbb{R}^{n} \backslash \bar{\Omega}$ имеет место асимптотическое разложсение интеграла (42):

$$
\begin{aligned}
I_{\varepsilon}^{\text {out }}(x)= & \frac{\left(2 \pi\left|B_{\xi}^{(1)}(\omega)\right|\right)^{(n-1) / 2}\left|S_{\xi}^{(1)}(x, \omega)\right|^{-1} \varepsilon^{(n+1) / 2}}{\operatorname{det}\left(\left|B_{\xi}^{(1)}(\omega)\right| S_{\eta \eta}^{(2)}(x, \omega)+\left|S_{\xi}^{(1)}(x, \omega)\right| B_{\eta \eta}^{(2)}(\omega)\right)^{1 / 2}} \\
& \times\left.\left(\varphi(\omega)+\varepsilon \varphi_{1}(x, \omega)+o(\varepsilon)\right) e^{-S(x, \omega) / \varepsilon}\right|_{\omega=\omega(x)}
\end{aligned}
$$

где $\varphi_{1}(x, \omega)=\widehat{L}(x, \omega) \varphi(\omega)$, а $\widehat{L}(x, \omega)$ - дифференииальный оператор второго порядка по переменным $\eta$ касательного пространства и первого порядка по трансверсальной переменной $\xi:$

$$
\widehat{L}(x, \omega)=\alpha(x, \omega)+a(x, \omega) \partial_{\xi}+\left(b(x, \omega), \nabla_{\eta}\right)+\frac{1}{2}\left(A(x, \omega) \nabla_{\eta}, \nabla_{\eta}\right) .
$$


Коэффициенты оператора $\widehat{L}(x, \omega)$ выражаются через моменты гауссова распределения $G_{x, \omega}$ в $\mathbb{R}^{n-1}$ с нулевым средним и коррелящионной матрищей:

$$
C(x, \omega)=\left|B_{\xi}^{(1)}(\omega)\right|^{-1}\left(\left|B_{\xi}^{(1)}(\omega)\right| S_{\eta \eta}^{(2)}(x, \omega)+\left|S_{\xi}^{(1)}(x, \omega)\right| B_{\eta \eta}^{(2)}(\omega)\right) .
$$

Обозначим через $\mathrm{M}_{x, \omega}$ математическое ожидание относительно гауссова распределения $G_{x, \omega}$. Тогда

$$
\begin{gathered}
A(x, \omega)=\mathrm{M}_{x, \omega} \eta^{\otimes 2}, \quad a(x, \omega)=\left|S_{\xi}^{(1)}(x, \omega)\right|^{-1}+\left|B_{\xi}^{(1)}(\omega)\right|^{-1} \mathrm{M}_{x, \omega} \operatorname{Tr}\left(B_{\eta \eta}^{(2)}(\omega) \eta^{\otimes 2}\right), \\
(b(x, \omega), z)=\mathrm{M}_{x, \omega} \operatorname{Tr}\left(\frac{1}{2\left|S_{\xi}^{(1)}(x, \omega)\right|}\left(\left(z \otimes\left(S_{\xi \eta}^{(2)}(x, \omega)+B_{\xi \eta}^{(2)}(x, \omega)\right)\right) \eta^{\otimes 2}\right)\right. \\
\left.+\left(\frac{1}{12} z \otimes\left(S_{\eta \eta \eta}^{(3)}+B_{\eta \eta \eta}^{(3)}\right)+\frac{1}{4\left|B_{\xi}^{(1)}(\omega)\right|} z \otimes\left(S_{\xi \eta}^{(2)} \otimes B_{\eta \eta}^{(2)}+S_{\eta \eta}^{(2)} \otimes B_{\xi \eta}^{(2)}\right)\right) \eta^{\otimes 4}\right) \\
\alpha(x, \omega)=\frac{S_{\xi \xi}^{(2)}(x, \omega)}{2\left|S_{\xi}^{(1)}(x, \omega)\right|}+\mathrm{M}_{x, \omega} \operatorname{Tr}\left(\frac{S_{\xi \xi}^{(2)} \otimes B_{\eta \eta}^{(2)}+S_{\eta \eta}^{(2)} \otimes B_{\xi \xi}^{(2)}}{2\left|S_{\xi}^{(1)}(x, \omega) B_{\xi}^{(1)}(\omega)\right|}\right. \\
+\left(\left(\frac{1}{24}\left(S_{\eta \eta \eta \eta}^{(4)}(x, \omega)+B_{\eta \eta \eta \eta}^{(4)}(\omega)\right)+\frac{\left(S_{\xi \eta \eta}^{(3)}(x, \omega)+B_{\xi \eta \eta}^{(3)}(\omega)\right) \otimes B_{\eta \eta}^{(2)}(\omega)}{4\left|B_{\xi}^{(1)}(\omega)\right|}\right.\right. \\
\left.\left.\left.+\frac{1}{8\left|B_{\xi}^{(1)}(\omega)\right|^{2}}\left(S_{\xi \xi}^{(2)}(x, \omega)+B_{\xi \xi}^{(2)}(\omega)\right)\left(B_{\eta \eta}^{(2)}(\omega) \otimes B_{\eta \eta}^{(2)}(\omega)\right)\right) \eta^{\otimes 4}\right)\right)
\end{gathered}
$$

где $\eta^{\otimes 2}$ и $\eta^{\otimes 4}$ - матрицы ранга 1 с независимыми случайными компонентами $\eta_{i} \eta_{j}$ и $\eta_{i} \eta_{j} \eta_{k} \eta_{l}(1 \leqslant i, j, k, l \leqslant n-1)$, имеющими гауссово распределение, заданное коррелящионной матрицей (46). Тензорным произведением матрии, $A=A_{i j k l}$ и $B=B_{i j k l}$ назьвается матрица $C=A \otimes B$ с коэффициентами $C_{i j m n}=\sum_{k, l} A_{i j k l} B_{k l m n}$, а следом $\operatorname{Tr}(A B)$ - сумма $\sum_{i, j, k, l} A_{i j k l} B_{k l i j}$.

Формулы (44)-(47) уточняют известные результаты [13] об асимптотике интегралов Лапласа в случае, когда $S_{v}^{(1)} \neq 0$ в точке условного минимума действия. Главньй член разложения (44) известен в более общей ситуации (см. [14], [15], а также [16] для интегралов Лапласа в банаховых пространствах).

Заметим также, что если $x \rightarrow \partial \Omega$, то $I_{\varepsilon}(x)=O\left(\varphi(x) \varepsilon^{n / 2}\right)$. С другой стороны, если $x \rightarrow \partial \Omega$, то $S_{\xi}^{(1)}(x, \omega) \rightarrow 0$; при этом условие (43) нарушается. Таким образом, асимптотическое разложение (47) справедливо в точках, отстоящих от $\partial \Omega$ на конечное расстояние.

В качестве примера рассмотрим асимптотику решения задачи Коши для параболического уравнения

$$
\left(-\varepsilon \partial_{t}+\frac{1}{2} \Delta+(V, \nabla)\right) u(x, t)=0
$$

Для простоты будем считать, что $\left.\operatorname{supp} u\right|_{t=0}=\Omega=\{x:|x| \leqslant R\}$ и $x, V \in \mathbb{R}^{2}$. Тогда асимптотика решения может быть найдена из явного интегрального представления решения с помощью леммы 2:

$$
u(x, t)=(2 \pi t \varepsilon)^{-1} \int_{\Omega} d y e^{-|x(t)-y|^{2} /(2 t \varepsilon)} u_{0}(y), \quad x(t)=x+V t .
$$


Если $x+V t \in \Omega=\{y:|y|<R\}$, то

$$
u_{\varepsilon}^{\mathrm{int}}(x, t)=\left.\left(1+\frac{\varepsilon t}{2} \Delta+O\left(\varepsilon^{2}\right)\right) u_{0}\right|_{x=x(t)}
$$

Если $x+V t \in \mathbb{R} \backslash \bar{\Omega}=\{y:|y| \leqslant R\}$, то $u_{\varepsilon}^{\mathrm{ext}}(x, t)=U(x(t), t)$, где

$$
\begin{aligned}
U(x, t)= & e^{-(|x|-R)^{2} /(2 \varepsilon t)}\left(\frac{t R}{2 \pi|x|}\right)^{1 / 2} \frac{1}{|x|-R}\left(u_{0}\left(\frac{R x}{|x|}\right)+\varepsilon U_{1}(x, t)+O\left(\varepsilon^{2}\right)\right), \\
U_{1}(x, t)= & \left(1-10 \frac{R}{|x|}+3\left(\frac{R}{|x|}\right)^{2}-\frac{t}{4(|x|-R)^{2}}\right) u_{0}\left(\frac{R x}{|x|}\right) \\
& +\frac{t}{(|x|-R)^{2}}\left(1+\left(\frac{R}{|x|}\right)^{1 / 2}\left(1-\frac{R}{|x|}\right)\left(\frac{x_{1}}{|x|} \partial_{y_{1}}+\frac{x_{2}}{|x|} \partial_{y_{2}}\right)\right. \\
& \left.+\left(\frac{R}{|x|}\right)^{3 / 2}\left(\frac{x_{1}}{|x|} \partial_{y_{2}}-\frac{x_{2}}{|x|} \partial_{y_{1}}\right)^{2}\right)\left.u_{0}(y)\right|_{y=R x /|x|^{*}}
\end{aligned}
$$

Нетрудно видеть, что

$$
\left.\left(\frac{x_{1}}{|x|} \partial_{y_{1}}+\frac{x_{2}}{|x|} \partial_{y_{2}}\right)\right|_{y=R x /|x|}
$$

- производная по нормали к границе, а

$$
\left.\left(\frac{x_{1}}{|x|} \partial_{y_{2}}-\frac{x_{2}}{|x|} \partial_{y_{1}}\right)^{2}\right|_{y=R x /|x|}
$$

- производная второго порядка по касательной к границе.

Разложения (48), (49) показьвают, что носитель главной части асимптотики решения переносится вдоль траекторий гамильтоновой системы $\dot{q}=H_{p}(q, 0), \dot{p}=-H_{q}(q, 0)$ с гамильтонианом $H(q, p)$, отвечающим параболическому уравнению (см. [7], [15]). В данном случае $H(q, p)=|p|^{2} / 2-(V, p)$ и эта система имеет вид $\dot{q}=p-V, \dot{p}=0, q(t)=x$, $p(0)=0$. Действие $S(x, t)$, определяющее экспоненциально убывающую часть решения, удовлетворяет уравнению Гамильтона-Якоби и является решением двухточечной вариационной задачи с граничными условиями: $q(t)=x, q(0) \in \partial \Omega(t), p(0) \perp \partial \Omega(t)$, $(p(0), n)>0$, где $n$ - внешняя нормаль к $\partial \Omega(t)$ в точке $q(0)$. Аналогичная ситуация имеет место и в случае уравнения Больцмана.

Используя метод построения асимптотики решений линейных уравнений туннельного типа в области больших уклонений от носителя начального условия [7], [15], [8], будем искать решение задачи Коши для уравнения (18) с финитньм и гладким на носителе $\Omega$ начальньм условием $p_{0}(v)$ в виде

$$
\rho_{\varepsilon}(v, t)=\int_{\Omega} d w G_{\varepsilon}(v, w, t) \rho_{0}(w),
$$

где $G_{\varepsilon}(v, w, t)$ - функция Грина $(38)$, отвечающая начальному условию $\rho_{0}(v)$.

Как было отмечено, для гамильтонианов, обладающих свойством $H_{p}^{(1)}(v, 0)=0$, самосогласованная динамика главной части решения $\rho_{\varepsilon}^{\text {int }}(v, t)$ оказывается тривиальной: $\rho_{\varepsilon}^{\text {int }}(v, t)=\rho_{0}(v)+O(\varepsilon), \Omega(t)=\Omega$. 
Теперь подстановка главного члена решения в уравнение (32) и лемма 2 дают уравнение Гамильтона-Якоби для действия в области больших уклонений:

$$
\partial_{t} S(v, w, t)+\int_{\Omega} d v_{2} \rho_{0}^{\mathrm{int}}\left(v_{2}\right) H\left(v-v_{2}, \nabla_{w} S(v, w, t)\right)=0
$$

а главньй член логарифмической асимптотики определяется решением этого уравнения в точке $w \in \partial \Omega, w=\arg \min S(v, w, t)$. Если выполнено условие невырожденности (29), то существует функция Лагранжа $\mathscr{L}(v, \dot{v})=p \dot{v}-\mathscr{H}(v, p), \dot{v}=\mathscr{H}_{p}^{(1)}(v, p)$, отвечающая гамильтониану

$$
\mathscr{H}(v, p)=\int_{\Omega} d v_{0} \rho_{0}\left(v_{0}\right) H\left(v-v_{0}, p\right)
$$

и по крайней мере для всех достаточно малых $t \leqslant T(v, w)$ существует решение двухточечной задачи для соответствующей системы Гамильтона (см. [3], [7]):

$$
\dot{v}(\tau)=\mathscr{H}_{p}^{(1)}(v(\tau), p(\tau)), \quad \dot{p}(\tau)=-\mathscr{H}_{v}^{(1)}(v(\tau), p(\tau)), \quad v(0)=v, \quad v(t)=w
$$

При этом главньй член логарифмической асимптотики равен

$$
S(v, t)=\min _{w \in \partial \Omega} S(v, w, t), \quad S(v, w, t)=\int_{0}^{t} d \tau \mathscr{L}(v(\tau), \dot{v}(\tau))
$$

Таким образом, имеет место теорема о логарифмической асимптотике решения задачи Коши для уравнения Больцмана.

ТЕОРемА 5. Пусть для гамильтониана $\mathscr{H}(v, p)$ выполнено условие невырохденности (29) и для распределения скачков скорости выполнены условия (22)-(24). Тогда главный член логарифмической асимптотики решения уравнения Больимана (18) в области больших уклонений равен $S(v, t)$, әде $S(v, t)$ - решение уравнения Гамильтона-Якоби

$$
\partial_{t} S(v, t)+\int_{\Omega} d v_{0} \rho_{0}\left(v_{0}\right) H\left(v-v\left(v_{0}, t\right), \nabla_{w} S(v, t)\right)=0
$$

а начальное и терминальное лагранжевы многообразия $\Lambda_{0} u \Lambda_{t}$, выделяющие единственное решение этого уравнения при всех достаточно малых $t$, имеют вид $\Lambda_{t}=\left\{v, p \in \mathbb{R}^{3}\right\}, \Lambda_{0}=\{v(0) \in \partial \Omega(t), p(0) \perp T \partial \Omega(t)\}$. 


\section{СПИСОК ЦИТИРОВАННОЙ ЛИТЕРАТУРЫ}

[1] Braun W., Hepp K. The Vlasov dynamics and its fluctuations in the $l / N$ limit of interacting classical particles // Comm. Math. Phys. 1977. V. 56. P. 101-113.

[2] Maslov V.P., Shvedov O. Yu. An asymptotic formula for the $N$-particle density function as $N \rightarrow \infty$ and violation of the chaos hypothesis // Russian J. Math. Phys. 1994. V. 2. № 2 . P. 217-234.

[3] Маслов В.П. Комплексные марковские цепи и континуальный интеграл Фейнмана. М.: Наука, 1980.

[4] Tanaka H. Probabilistic treatment of the Boltzmann equation of Maxwellian molecules // Z. Wahrscheinlichkeitstheorie Verw. Gebiete. 1978. V. 46. P. 67-105.

[5] Лукшин Л.В. Стохастические алгоритмы математической теории пространственно неоднородного уравнения Болцмана // Матем. моделирование. 1989. Т. 1. № 2. С. 151-160.

[6] Арсеньев А. А. Лекции о кинетических уравнениях. М.: Наука, 1992.

[7] Маслов В. П. Асимптотические методы и теория возмущений. М.: Наука, 1988.

[8] Маслов В.П. Асимптотика решений $N$-частичных уравнений Колмогорова-Феллера и асимптотика решения уравнения Больмана в области больших уклонений // Матем. заметки. 1995. Т. 58. № 5. С. 694-709.

[9] Бэдсел И., Ленгдон А. Физика плазмы и численное моделирование. М.: Энергоиздат, 1989.

[10] Гихман И.И., Скороход А. В. Введение в теорию случайных процессов. М.: Наука, 1977.

[11] Чеботарёв А. М. Достаточные условия регулярности скачкообразных марковских процессов // Теория вероятн. и ее применения. 1988. Т. 33. № 1. С. 25-39.

[12] Ландау Л. Д. Кинетические уравнения в случае кулоновского взаимодействия. Т. 1. М.: Наука, 1969.

[13] Федорюк М. В. Метод перевала. М.: Наука, 1977.

[14] Skinner L. A. Note on the asymptotic behavior of multidimensional Laplace integrals // SIAM J. Math. Anal. 1980. V. 11. № 5. P. 911-917.

[15] Маслов В. П., Фроловичев С. М., Черных С. И. Точная асимптотика больших уклонений решений граничных задач для дифференциальных уравнений // Докл. АН СССР. 1987. T. 296. № 2. C. $275-279$.

[16] Питербарг В.И., Фатафлов В. Р. Метод Лапласа для вероятностных мер в банаховых пространствах // УМН. 1995. Т. 50. №6. С. 58-150.

Московский государственный университет им. М.В. Ломоносова

E-mail: alex@cheb.phys.msu.su 